Corrected: Publisher Correction

ARTICLE

https://doi.org/10.1038/s42005-019-0228-3 OPEN

\title{
The effect of introducing antibiotics into organic light-emitting diodes
}

Hassan Hafeez (10 1,12, P. Justin Jesuraj ${ }^{1,12}$, Dong Hyun Kim¹,12, Jong Chan Lee ${ }^{1}$, Jun Young Shin', Sang Ho Rhee ${ }^{2}$, Won Ho Lee ${ }^{1}$, Dae Keun Choi ${ }^{1}$, Jun Hwan Cha ${ }^{1}$, Chang Min Lee ${ }^{1}$, Chul Hoon Kim ${ }^{3 \star}$, Janardan Lamichhane ${ }^{4}$, Anaya Pokhrel (10 4, Tae-Su Kim ${ }^{4}$, Jae Kyung Sohng ${ }^{4}$, Hyung Joong Yun ${ }^{5}$, Jong Bae Park (10 ${ }^{6}$, Hee-Suk Chung (1) ${ }^{6}$, Tae-Sung Bae ${ }^{6}$, Sang Geul Lee (10 ${ }^{7}$, Hyun-Woo Park ${ }^{8}$, Kwun-Bum Chung ${ }^{8}$, Aeran Song ${ }^{8}$, Jang Hyuk Kwon (D) ${ }^{9}$, Hyeong Woo Bae ${ }^{9}$, Yong-Cheol Kang ${ }^{10}$, Juyun Park ${ }^{10}$, Myungkwan Song ${ }^{11}$, Chang-Su Kim ${ }^{11}$ \&

Seung Yoon Ryu (iD ${ }^{1 *}$

The quest to improve the performance of organic light-emitting diodes (OLEDs) has led to the exploration of new materials with properties like interfacial dipole, excitons generation, and bandgap alignment. Here, we exploit these strategies by investigating the interaction of the antibiotic ampicillin with a widely used optoelectronic material, to fabricate state-of-theart OLEDs. The charge distribution on the ampicillin molecule facilitates the generation of an interfacial dipole with a large magnitude. The optimum fusion of the two materials provides an enhanced bandgap alignment, charge balance and $\mathrm{J} / \mathrm{H}$-aggregated excitons. Values of current efficiency $\left(120 \mathrm{cdA}^{-1}\right)$, external quantum efficiency $(\sim 35 \%)$ and power efficiency $\left(70 \mathrm{ImW}^{-1}\right)$ are demonstrated. The cross-evaluation of performance with penicillin devices indicates the significance of ampicillin's specific molecular structure in improving performance. The detailed investigations demonstrate that ampicillin has superior optoelectronic properties with high potential to contribute extensively in OLEDs and photovoltaics.

\footnotetext{
${ }^{1}$ Division of Display and Semiconductor Physics, Display Convergence, College of Science and Technology, Korea University Sejong Campus 2511 Sejong-ro, Sejong City 30019, Republic of Korea. ${ }^{2}$ Department of Information Communication \& Display Engineering, Division of Mechanical and ICT Convergence Engineering, Sun Moon University, 221, Sunmoon-ro, Tangjeong-myeon, Asan, Chungnam 31460, Republic of Korea. ${ }^{3}$ Department of Advanced Materials Chemistry, College of Science and Technology, Korea University Sejong Campus 2511 Sejong-ro, Sejong City 30019, Republic of Korea. ${ }^{4}$ Department of Life Science and Biochemical Engineering, SunMoon University, 70 Sunmoon-ro 221, Tangjeong-myeon, Asan-si, Chungnam 31460, Korea. 5 Advanced Nano Surface Research Group, Korea Basic Science Institute (KBSI), 169-148 Gwahak-ro, Daejeon 34133, Republic of Korea. ${ }^{6}$ Jeonju Center, Korea Basic Science Institute (KBSI), 20, Geonji-ro, Deokjin-gu, Jeonju, Jeollabuk-do 54907, Republic of Korea. ${ }^{7}$ Daegu Center, Korea Basic Science Institute (KBSI), 80, Daehakro, Buk-gu, Daegu 41566, Republic of Korea. ${ }^{8}$ Division of Physics and Semiconductor Science, Dongguk University, Seoul 04620, Republic of Korea.

${ }^{9}$ Department of Information Display, Kyung Hee University, Dongdaemun-gu, Seoul 130-701, Republic of Korea. ${ }^{10}$ Department of Chemistry, Pukyong National University, 45 Yongso-Ro, Nam-gu, Busan 48513, Republic of Korea. ${ }^{11}$ Surface Technology Division, Korea Institute of Materials Science (KIMS) Changwon, Changwon 51508, Republic of Korea. ${ }^{12}$ These authors contributed equally: Hassan Hafeez, P. Justin Jesuraj, Dong Hyun Kim.

*email: chulhoon@korea.ac.kr; justie74@korea.ac.kr
} 
O ver the past five decades, the semiconductor industry has provided infinite scope for improvement. Many inorganic materials and devices were investigated, leading to developments in artificial intelligence (AI)-such as Alphago ${ }^{1,2}$. Recently, the rate of progress in inorganic materials has saturated, and Moore's law has probably approached its limit. ${ }^{3}$ To overcome these limitations, we ought to incorporate new candidates such as antibiotics and biomaterials instead of Silicon or other conventional elements, to form the next generation of devices. ${ }^{4,5}$ For organic optoelectronics such as organic light-emitting diodes (OLEDs) and photovoltaics (OPVs), Poly(3,4-ethylenedioxythiophene): poly(styrenesulfonate) (PEDOT:PSS) is one of the most widely used materials for various purposes, such as hole transporting layer (HTL), anode buffer layer, and transparent conducting electrode. ${ }^{6-8}$ Recently, significant endeavors and effective approaches have been demonstrated for enhancing the conductivity of PEDOT:PSS. ${ }^{9}$ For example, Zhang et al. ${ }^{10}$ demonstrated that the addition of glycerol or sorbitol could increase the conductivity of the PEDOT:PSS by two orders of magnitude. Ouyang et al. ${ }^{11}$ utilized meso-erythritol or dimethyl sulfoxide to modify PEDOT:PSS properties. Rivney et al. ${ }^{12}$ showed that the addition of ethylene glycol improves conductivity and generates a controlled aggregation (such as a $\mathrm{J}$ or $\mathrm{H}$ aggregation, with marginally tighter $\pi$-stacking) to empower electronic and ionic transport for attaining energy efficient devices. Wang et al. $^{13}$ also reported a parallel J-aggregation in poly(diallyldimethylammonium) chloride (PDDA) with the help of amine group $\left(-\mathrm{NH}_{2}\right)$ and -PSS, which acted as a bridge for faster exciton transfer between quantum dots. In search of a proper addition to PEDOT:PSS to make it more favorable, we investigated the chemical, optical, and electronic properties of ampicillin and studied its interaction with PEDOT:PSS.

Ampicillin is most widely used as an antibiotic for animal health (like penicillin is for human health) ${ }^{14-17}$ because of its stability in acidic conditions. Even though, most research on optoelectronic devices demonstrated vertical (orthogonal) dipole formation; ${ }^{18-20}$ the spatial orientation of the generated horizontal (or inclined) interfacial dipole in ampicillin molecule with respect to changes in $\mathrm{pH}$ was recently simulated by Malloci et al. ${ }^{21}$ The primary, secondary, and tertiary (cyclic amide in lactam ring) amine along with the carbonyl group $(-\mathrm{C}=\mathrm{O})$ present in ampicillin's structure provide a specific charge distribution over the molecule. ${ }^{21}$ This resulted in an interfacial dipole with a larger magnitude (30.28 Debye) $)^{21,22}$ as compared with the dipole from other amine-based molecules such as polyethyleneimine (PEI) $(1.3 \text { Debye })^{23}$ and even penicillin (5.99 Debye). ${ }^{24}$ The utilization of amine-based molecules including biomaterials and amino acids have been previously reported to alter the work function $(\varphi)$ of the layers/substrates owing to the generation of interfacial dipole for various optoelectronic and OPV applications. ${ }^{25-28}$ Mostly, a reduction in the $\varphi$ of the indium tin oxide (ITO) substrates was achieved by treatment with the amine-based molecules having a dipole away from the ITO. ${ }^{26,28}$ In addition, Kotagiri et al. ${ }^{29}$ highlighted the emission properties of ampicillin as an efficient fluorescent agent and the energies of the aggregated molecules with respect to their arrangement could be expressed by Brick theory. ${ }^{30}$ So, we chose ampicillin to be incorporated in optoelectronics because materials with such interfacial dipoles, whether utilized as thin films ${ }^{18,19,31}$ or in bulk form, ${ }^{20}$ has proven to be beneficial for OLED's performance.

In terms of enhancements in device performance of green OLEDs to date, Huang et al. ${ }^{32}$ reported an external quantum efficiency (EQE) of $32.5 \%$ and a current efficiency (CE) of 117.5 $\mathrm{cd} \mathrm{A}^{-1}$ in a tandem structure. $\mathrm{Xu}$, et al. ${ }^{33}$ reported a maximum EQE of $36.85 \%$ and $\mathrm{CE}$ of $135.74 \mathrm{~cd} \mathrm{~A}^{-1}$ also in a tandem structure. In addition, thermally activated delayed fluorescence devices have demonstrated an EQE of $35-37.8 \%$ owing to the horizontal dipole molecular orientation and intramolecular charger transfer processes. ${ }^{34-36}$ Helander et al. ${ }^{37}$ utilized a chlorinated ITO in single unit OLED to present an EQE of 29.1\% owing to the tuning of the anode $\varphi$ (charge balance strategy) without any modifications in the molecular orientation of host/ dopant or light out-coupling. Meanwhile, Kim, et al. ${ }^{38}$ experimentally demonstrated an EQE of $30 \%$ by horizontal dipole orientation and theoretically analyzed that a maximum of $46 \%$ EQE could be achieved in ITO-based single unit OLEDs.

In this work, we demonstrate a substantial increment in the device performance of a single-unit OLED without any outcoupling aid (EQE 35.1\%). The performance was boosted by horizontal/inclined interfacial dipole, charge balance and extra J/ $\mathrm{H}$-aggregated exciton generation, which was achieved by the contribution of ampicillin in PEDOT:PSS (ampicillin-PEDOT: PSS). For further evaluation, OLED devices with similar concentrations $(25-75 \%)$ were also fabricated by the addition of penicillin, which has an identical chemical structure, but the only difference is the absence of primary $-\mathrm{NH}_{2}$. This provided a progressive insight about the specialty and significance of the ampicillin's structure and specific interfacial dipole characteristics in the enhancement of OLED's device performance. The physical, chemical, optical, and electronic properties, along with the concentration- and time-dependent interactions of ampicillin with PEDOT:PSS, leading to its role in optoelectronic performance, was investigated.

\section{Results}

Electrical and optical properties of ampicillin-PEDOT:PSS. Figure 1a shows the schematic of the OLED device (ITO/(0-75\%) ampicillin-PEDOT:PSS/N,N-bis-(1-naphthyl)- $N, N^{\prime}$-diphenyl-1,1'biphenyl-4,4'-diamine (NPB)/Tris(4-carbazoyl-9-ylphenyl)amine (TCTA) $/ 4,4^{\prime}$-Bis $(N$-carbazolyl)-1,1'-biphenyl (CBP)+Tris[2-phenylpyridinato-C2,N]iridium(III) (Ir(ppy) $\left.)_{3}\right) / 2,2^{\prime}, 2^{\prime \prime}-(1,3,5$-benzinetriyl)-tris(1-phenyl-1-H-benzimidazole) (TPBi)/lithium fluoride (LiF)/aluminum (Al)) layout with the bandgap alignments of layers as shown in Fig. 1b. The carrier injection barrier heights (i.e., difference in Fermi levels $\left(\mathrm{E}_{f}\right)$ between layers) determine the performance of an optoelectronic device. ${ }^{39}$ The valence electronic energy levels of ampicillin-PEDOT:PSS were analyzed using ultraviolet photoelectron spectroscopy (UPS as shown in Fig. 1c) by depositing on a clean Au surface. The analysis of the ampicillin-PEDOT:PSS layers revealed a decrease in $\mathrm{E}_{f}$ with an increase in amount of ampicillin. At 0\% (only PEDOT:PSS), the $\varphi$ was found to be $4.74 \mathrm{eV}$, which decreased to $4.10 \mathrm{eV}$ at $25 \%$ ampicillin addition. The $\varphi$ values of the ampicillin-PEDOT:PSS further decreased to 3.75 and $3.72 \mathrm{eV}$, with the addition of $40 \%$ and $75 \%$ concentrations, respectively. Moreover, we compared the measured $\varphi$ using UPS with the one analyzed by kelvin probe force microscopy (KPFM) (Supplementary Fig. 1). Even though the surface $\varphi$ obtained by the KPFM was slightly higher owing to different nature of the analysis (atmospheric conditions might have caused some contamination/oxidation), ${ }^{40}$ the overall trend of decrease in $\varphi$ with the addition of ampicillin was found to be in agreement with the UPS measurements.

We attribute this decrease in $\varphi$ for ampicillin-PEDOT:PSS layers to the shift of $\mathrm{E}_{f}$ towards the lowest unoccupied molecular orbital owing to the generation of an opposite interfacial dipole directed towards the anode. This indicated a concentrationdependent interaction of the ampicillin-PEDOT:PSS, which was analyzed using X-ray photoelectron spectroscopy (XPS) as shown in Fig. 1d. The carboxylic acid-related photoelectronic peak in C1 s profile $(-\mathrm{COOH} \text { at } 288.1 \mathrm{eV})^{41,42}$ detected with an addition of $25 \%$ confirmed the presence of ampicillin in the ampicillin- 


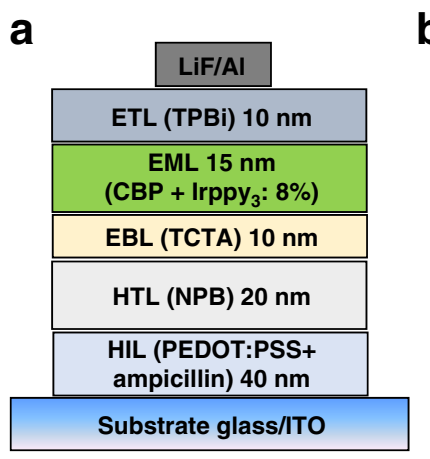

b

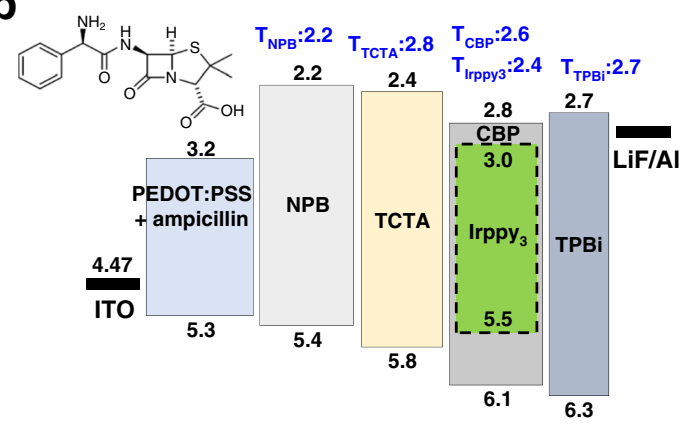

C

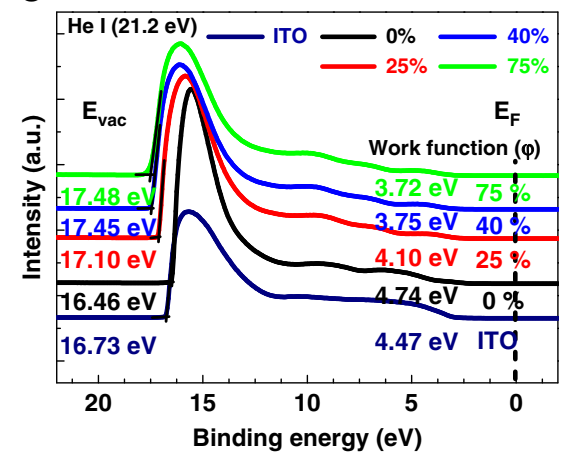

d

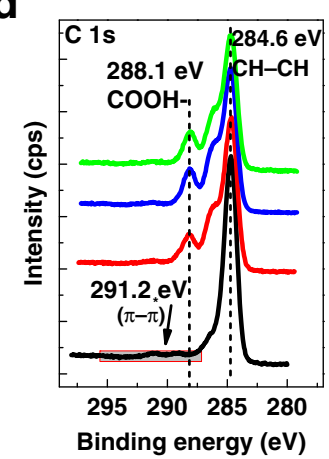

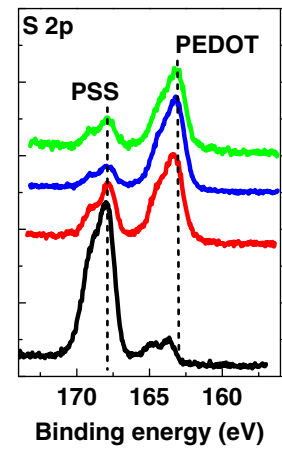

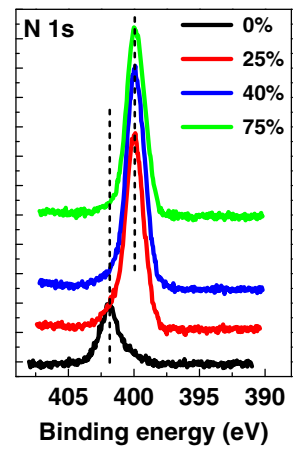

e

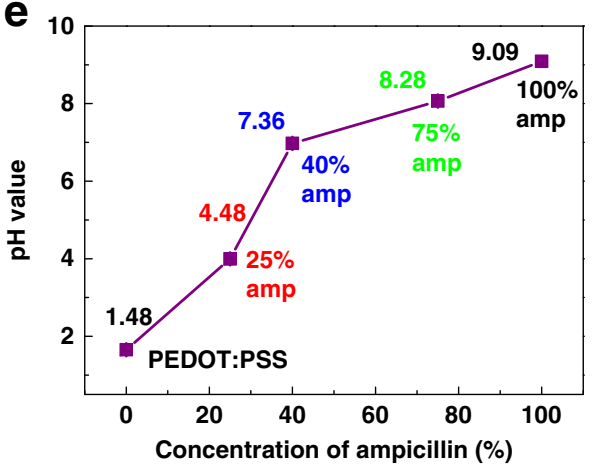

Fig. 1 Electrical and optical properties of (0-75\%) ampicillin-PEDOT:PSS layers. a Schematic of the fabricated organic light-emitting diodes (OLED) devices with (0-75\%) ampicillin in PEDOT:PSS. b Bandgap alignment of the utilized layers in the device structure along with the triplet energies of the layers and the chemical structure of ampicillin. c Ultra-violet photoelectron spectroscopy (UPS) analysis with secondary cutoff regions of indium tin oxide (ITO)/ (0-75\%) ampicillin-PEDOT:PSS. $\mathbf{d}$ X-ray photoelectron spectroscopy (XPS) analysis of the PEDOT:PSS layers showing - $\mathrm{COOH}$ and $-\mathrm{N}$ confirming the presence of ampicillin's carbonyl and $-\mathrm{NH}_{2}$ groups, respectively. e $\mathrm{pH}$ analysis (standard deviation from the mean, obtained average from five number of measurements) of different concentrations of ampicillin-PEDOT:PSS solutions showing the shift from acidic $(\mathrm{pH}<7)$ to alkaline $(\mathrm{pH}>7)$ nature of solutions with increase in concentration of ampicillin

PEDOT:PSS. The intensity of the peak (at $400 \mathrm{eV})^{41,42}$ of $N$ at $0 \%$ increased when the ampicillin concentration rose to $25 \%$ and so on, which refers to the presence of $-\mathrm{NH}_{2}$ from the ampicillin in ampicillin-PEDOT:PSS. We also observed a small $\mathrm{N} 1 \mathrm{~s}$ peak for PEDOT:PSS material which does not contain $\mathrm{N}$ in its molecular structure as reported before. ${ }^{43,44} \mathrm{We}$ speculate that the peak was due to contamination from the atmosphere during the transfer of samples from the preparation facility to the XPS analysis tool. We prepared several samples at different conditions including glove box, open air followed by placement in the loading chamber, however, we still observed this N $1 \mathrm{~s}$ peak for PEDOT:PSS.43,44 On analyzing the S-2p profile, a large -PSS peak was encountered for $0 \%$, which was reduced in intensity for $25 \%$ film, and almost disappeared in $40 \%$, indicating that the majority of -PSS group in PEDOT:PSS was attached to $-\mathrm{NH}_{2}$ side chain. The shifting realized in ITO peaks (Supplementary Fig. 1) also corroborated the lowered $\varphi^{45}$ of ampicillin-PEDOT:PSS as supported with UPS analysis, where the overall $\varphi$ of the system reduced with increased addition of ampicillin. ${ }^{18,19,31}$

In addition to the changes in $\varphi$, charge transport properties are highly affected by the structure (amorphous/crystalline) and surface morphology. Surface morphology and structure of the ampicillin-PEDOT:PSS were analyzed using atomic force microscopy (AFM), x-ray diffraction (XRD), and high-resolution transmission electron microscopy (HR-TEM), as illustrated in (Supplementary Fig. 1). However, we did not observe any major changes in surface morphology (except 25\% ampicillin-PEDOT: PSS) and any crystallinity from XRD and HR-TEM analysis was not detected. Absorbance/transmittance analysis was also in accordance with the slight variations detected in the surface morphology of the layers (Supplementary Fig. 2). Hence, the analysis indicates that the interaction of ampicillin in PEDOT:PSS purely depends on volume concentrations.

Concentration-dependent chemical interactions of ampicillin. The ampicillin chemical structure ${ }^{46,47}$ contains an electrophile $-\mathrm{NH}_{2}$ (primary amine) group, secondary amine, nucleophile -C $=\mathrm{O}$, and a lactam ring (tertiary amine) (Supplementary Fig. 3). The amine side chain of ampicillin gives it stability in acidic conditions $(\mathrm{pH} \sim 4.48(25 \%))$ but, makes ampicillin unstable in alkaline $(\sim 7.36(40 \%), \sim 8.28(75 \%))$ conditions by opening up the lactam ring. 47,48 An increase in $\mathrm{pH}$ was analyzed with an increase in concentration of ampicillin in PEDOT:PSS (Fig. 1e). At $25 \%$ concentration, the acidic nature of the solution permits the existence of ampicillin's stable monomer form. The asymmetry in this stable ampicillin monomer charge distribution owing to the presence of primary $-\mathrm{NH}_{2}$ and $-\mathrm{C}=\mathrm{O}$, results in the generation of a net interfacial dipole with a large magnitude of

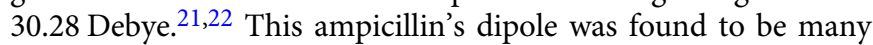
orders higher than other amine-based molecules (Supplementary Fig. 3) such as PEI, which has a dipole of 1.3 Debye. ${ }^{23}$ With just the absence of primary amine, such as in penicillin structure, the dipole moment was also greatly reduced to 5.99 Debye. $^{24}$ This indicates that unlike other amine-based molecules, the specific molecular structure of ampicillin is a crucial aspect, which could significantly contribute in the resulting device performance. The spatial orientation of the generated dipoles in ampicillin 


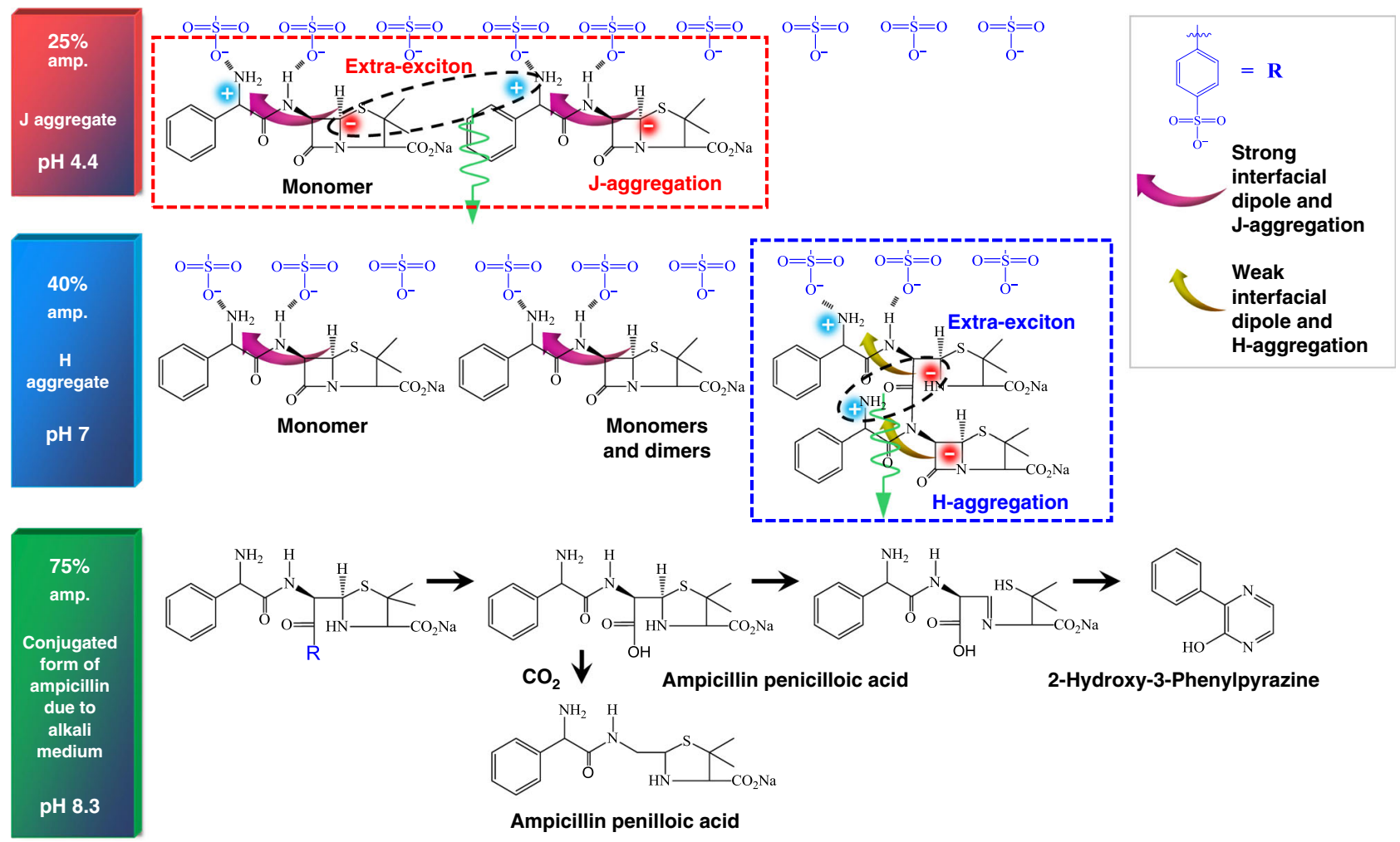

Fig. 2 Schematic of the chemical interaction of ampicillin (0-75\%) with PEDOT:PSS. The 25\% ampicillin monomers attached with PEDOT:PSS in a horizontal fashion contribute extra excitons by J-aggregation. With $40 \%$ ampicillin addition (due to the start of polymerization) some of the monomers formed dimers in a more perpendicular manner, which resulted in the generation of $\mathrm{H}$ aggregations. With $75 \%$ addition ampicillin became unstable and decomposed to conjugated forms owing to high $\mathrm{pH}$

molecules with respect to $\mathrm{pH}$ was simulated before (Supplementary Fig. 3). ${ }^{21}$ Based on the simulated dipole characteristics, the interaction of $25 \%$ ampicillin-PEDOT:PSS could be demonstrated as shown in Fig. 2. The interaction was experimentally analyzed using high-performance liquid chromatography (HPLC) (Supplementary Fig. 4), which indicated a reduction in -PSS peak together with an increment in ampicillin peak. This was coherent with the peak variations analyzed using XPS (Fig. 1d). Hence, from XPS and HPLC analysis, it was acquired that -O group from $-\mathrm{SO}_{3}$ molecules of -PSS interacted with $-\mathrm{NH}_{2}$ and $-\mathrm{H}$ groups of ampicillin monomers through hydrogen bonding (Fig. 2). The ampicillin molecules attached with PEDOT:PSS chain, which formed a head-to-tail arrangement to induce J-aggregation also cross-examined by S-parameter analysis of molecular orientation (Supplementary Fig. 5) ${ }^{49-51}$ Owing to the parallel location of primary, secondary, and tertiary amines in the main chemical structure of ampicillin (Supplementary Fig. 3), the placement might be beneficial to form a horizontal (parallel) interfacial dipole with PEDOT:PSS, as shown in Fig. 2.

Evaluation and analysis of $\mathbf{J} / \mathrm{H}$-aggregated excitons. Figure $3 \mathrm{a}, \mathrm{b}$ shows the mechanism of the efficiency contribution in the device by the horizontal/inclined interfacial dipole and the generated $\mathrm{J} / \mathrm{H}$ aggregations. The changes in energy of the resulting molecular arrangement owing to horizontal and inclined dipoles would differ (Fig. $3 c)^{52}$ and the detailed mechanism of $\mathrm{J} / \mathrm{H}$ aggregations is demonstrated in Supplementary Fig. 5. It is to be noted, that in previous reports, other amine-based layers such as PEI, ${ }^{19,23} \mathrm{PEI}$ : PSS $^{18}$ and polyallylamine ${ }^{19}$ lowered the $\varphi$ of the cathode and reduced the electron transport barrier. However, in our work, the high magnitude of the dipole by ampicillin, lowered the $\varphi$, which suppressed the hole injection owing to the dipole direction towards the ITO anode. Also, the hole injection was relatively higher for 25\% ampicillin-PEDOT:PSS as compared with $40 \%$, which could be due to the orientation of the inclined interfacial dipole that suppressed hole injection more than the horizontal dipole. $^{18-20}$ We examined this effect of interfacial dipole in different concentrations of ampicillin using transport stability analysis through forward $(0-8 \mathrm{~V})$ and reverse $(8-0 \mathrm{~V})$ applied voltage (Fig. 3d). A similar current density trend observed for $0 \%$ (PEDOT:PSS) layer revealed a negligible charge trapping/detrapping phenomena. However, with an addition of ampicillin (e.g., $40 \%$ ), the current density difference between forward and reverse bias indicated that ampicillin addition in PEDOT:PSS induced a charge trapping/de-trapping or accumulation, which might be owing to the interfacial dipole (Fig. 3d). ${ }^{18-20}$ Further, the expected efficiency contribution of this high magnitude dipoles and $\mathrm{J} / \mathrm{H}$ aggregations was analyzed by the obtained $\mathrm{EQE}$ from heterojunction devices (Fig. 3e), which were fabricated without a proper EML. Here, the pristine (without ampicillin) PEDOT:PSS devices presented a very low efficiency of $\sim 0.5 \%$. The efficiency of the devices was increased to $\sim 4-6 \%$ with the addition of ampicillin, which indicates that even without EML, light emission was obtained. This emission by the ampicillinPEDOT:PSS layer in heterojunction devices could be attributed to the additional/extra $\mathrm{J} / \mathrm{H}$-aggregated excitons generated due to the ampicillin's molecular dipole. The mechanism for the generation of such aggregations with the addition of dipole in the $-\mathrm{NH}_{2}$ based dyes has been systematically reviewed by Brixner et al. ${ }^{53}$ Also, the J-aggregations were found to be preferable where less electrical loss and better optical model was required. ${ }^{38}$ Moreover, Qin et al. ${ }^{54}$ also demonstrated that an aggregates-based single thin film was utilized as both HTL and EML purposes where 
a

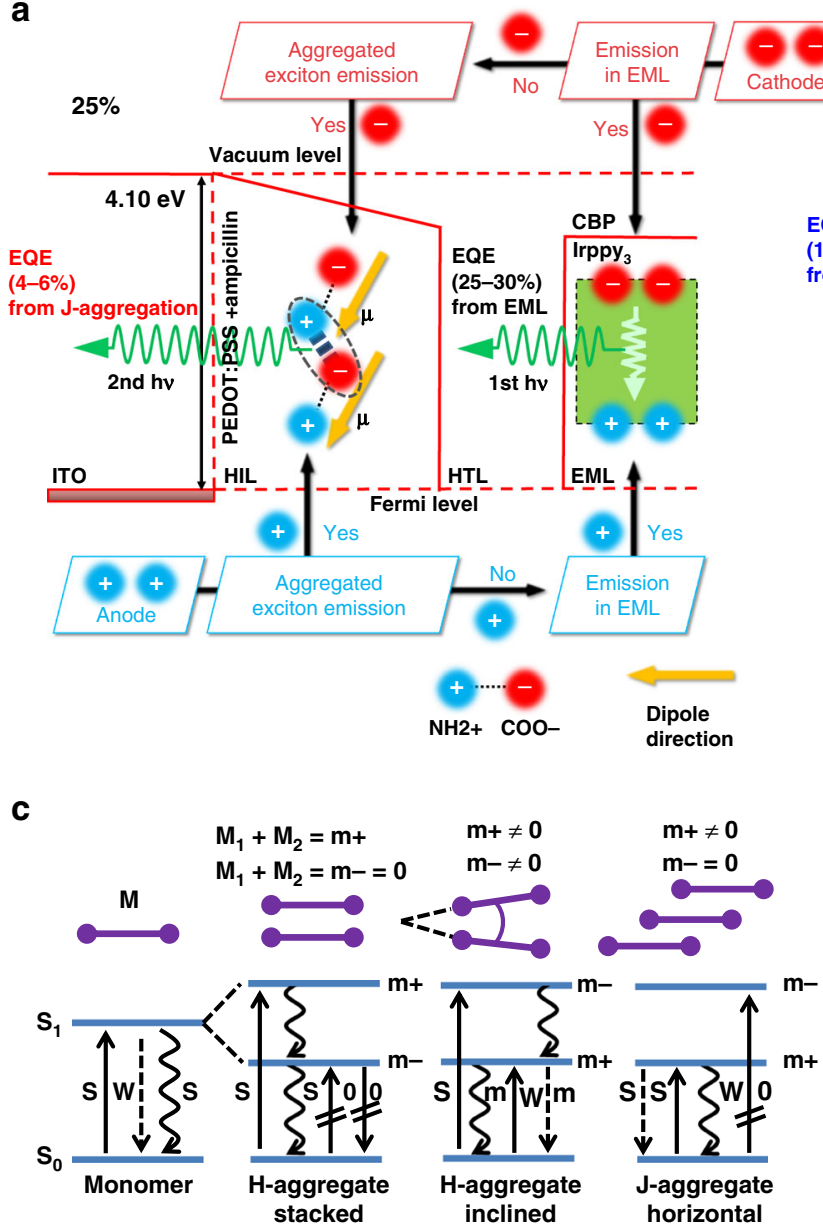

e

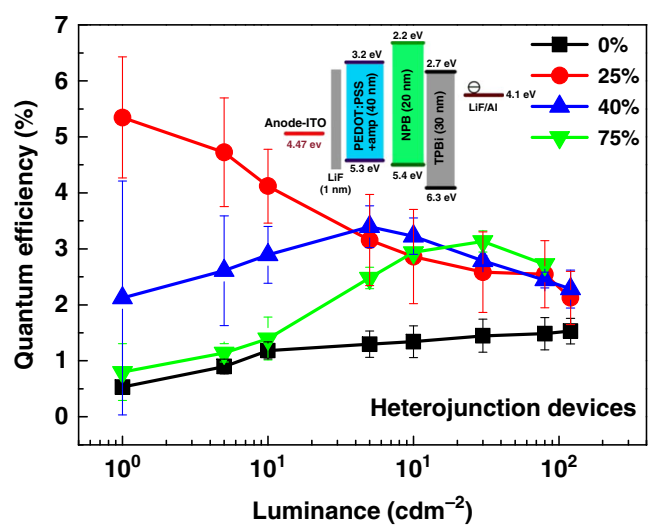

b
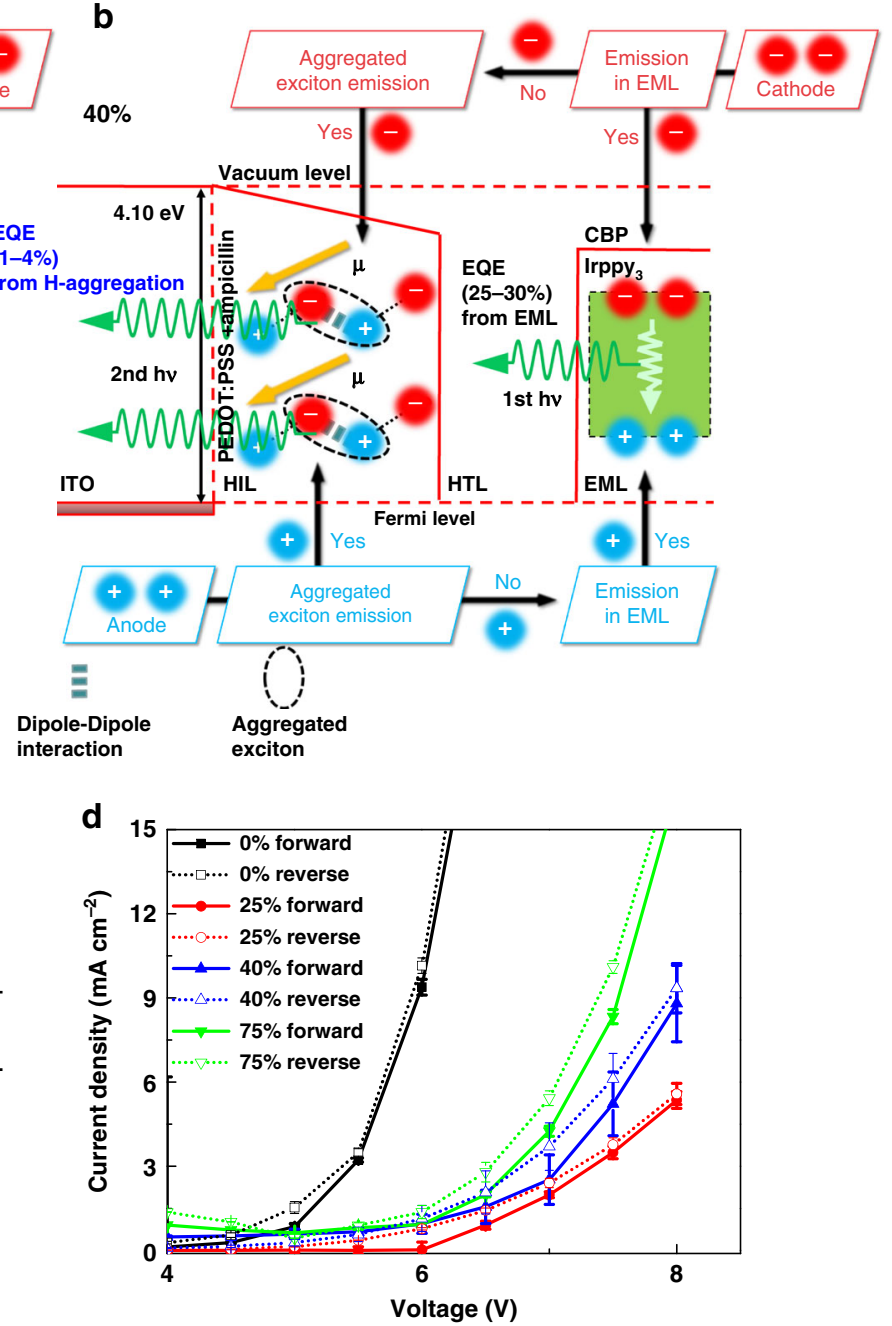

f

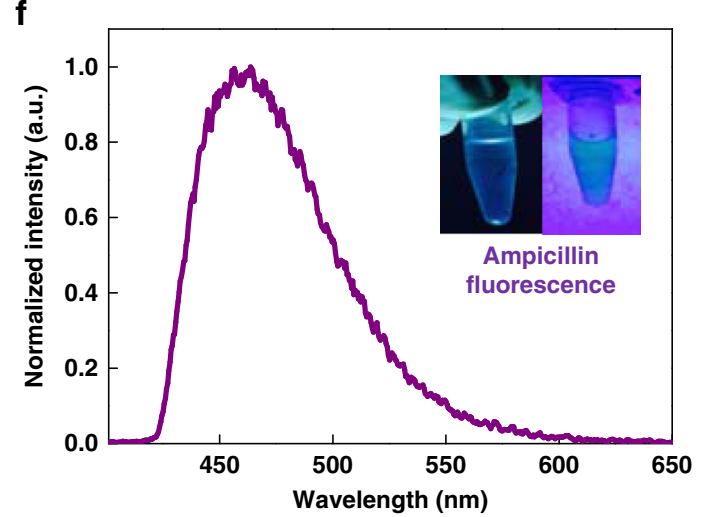

Fig. 3 Mechanism of the efficiency contribution by J/H-aggregated excitons. $\mathbf{a}, \mathbf{b}$ Schematic of the overall efficiency contribution by $\mathrm{J} / \mathrm{H}$-aggregated excitons, emission layer (EML) along with the interfacial dipole orientations for (a) $25 \%$ and (b) $40 \%$ ampicillin addition. c demonstrates the excitation types involved in relation to monomers, horizontal (J-aggregate)/inclined (H-aggregates). Where, $\mathrm{S}$ represents absorption, $\mathrm{W}$ for fluorescence, wavy arrows for internal conversion, and crossed lines for forbidden transitions, $\mathrm{M}$ for transition dipole moments and $\mathrm{m}$ for excitonic states ${ }^{52}$. $\mathbf{d}$ Current densityvoltage (J-V) of ampicillin-PEDOT:PSS layers (0-75\%) under forward and reverse applied potential (standard deviation from the mean, obtained by averaging five number of measurements is shown as error bars). e External quantum efficiency (EQE) analyzed (errors are standard deviation from the mean, obtained average eight number of measurements) for heterojunction devices without the addition of EML. $\mathbf{f}$ showing the camera images of ampicillin fluorescence under UV and steady state photoluminescence (PL)

$\sim 3.5 \% \mathrm{EQE}$ was obtained. Therefore, the obtained results from the heterojunction devices (Fig. 3e) provided a strong evidence that a boosted emission by $\mathrm{J} / \mathrm{H}$-aggregated excitons could be possible and expected in the full OLED device structure. The overall summary of the mechanism is presented in Fig. 3a, b where two different emissions could be concluded: one at the EML with 25-30\% EQE owing to the charge (hole-electron) recombination; the second at the ampicillin-PEDOT:PSS layer 

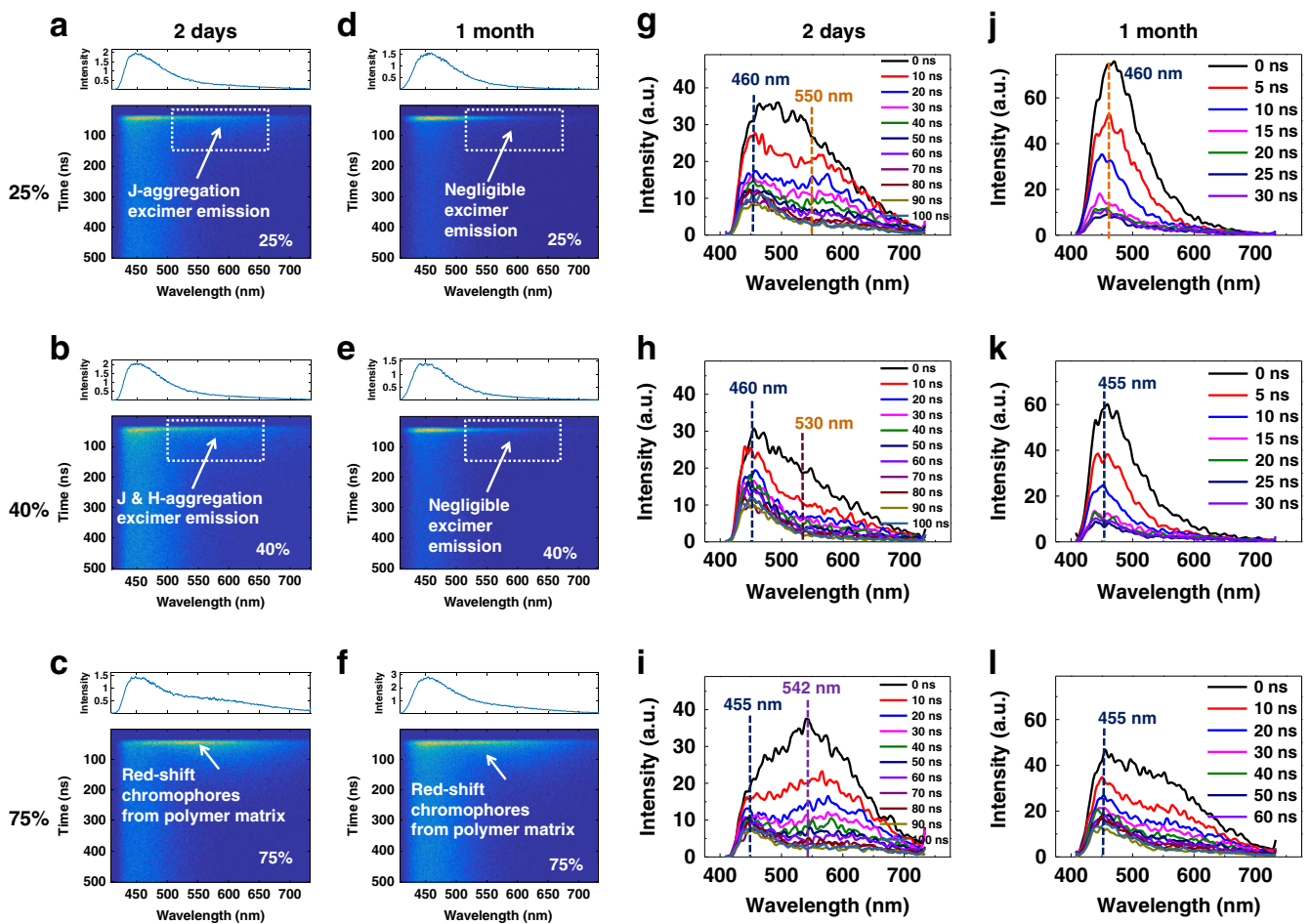
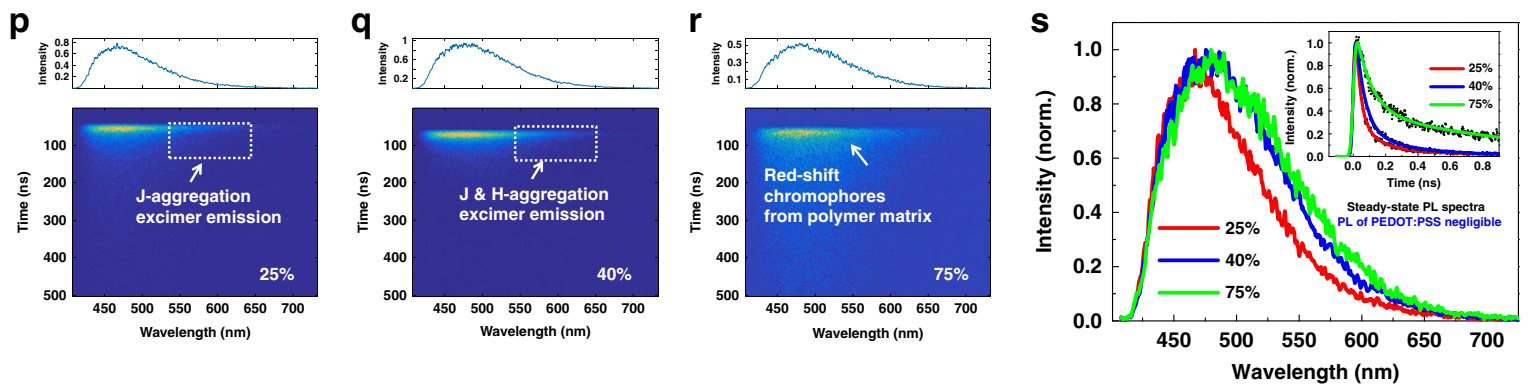

Fig. 4 Time-resolved photoluminance (TRPL)/Time-resolved area normalized emission spectrum (TRANES) analysis of (0-75\%) ampicillin-PEDOT:PSS layers on indium tin oxide (ITO) and fused silica substrate. a-c TRANES analysis for 2 days fresh ampicillin-PEDOT:PSS. a J-aggregation emission at about 500-750 nm for 25\% ampicillin-PEDOT:PSS on ITO. b J and H aggregation emission for 40\% ampicillin-PEDOT:PSS on ITO. c Excimer emission for 75\% ampicillin-PEDOT:PSS on ITO showing chromophores emission for decomposed forms of ampicillin. d-f TRANES analysis of 1-month-old ampicillinPEDOT:PSS samples showing negligible excimer emissions. Corresponding TRANES for each concentration of $\mathbf{g}$-i 2-days fresh $\mathbf{j}$-I 1-month-old ampicillinPEDOT:PSS. $\mathbf{m}$-o Normalized intensity comparison for 2-days and 1-month (25-75\%) ampicillin-PEDOT:PSS for 0 ns exciton lifetime. p-r TRANES mapping images of intensity and lifetime variation of PEDOT:PSS films with ampicillin addition deposited on fused silica. $\mathbf{s}$ solid-state emission spectra of PEDOT:PSS films with various concentrations (25-75\%) of ampicillin deposited on fused silica. Inset depicts the exciton lifetime (TRPL) of corresponding concentrations

with $~ 4-6 \%$ EQE in case of $25 \%$, and $1-4 \%$ for $40 \%$ addition of ampicillin. These excitons were also confirmed by the picosecond time-resolved photoluminescence (TRPL) and time-resolved area normalized emission spectrum (TRANES), and are discussed in the coming section. Here, the out-coupling factor could be overlooked because we did not observe any significant changes in the analyzed angle dependent optical spectra even with various backing temperatures and substrates (data not shown).

Another significant effect of ampicillin is the fluorescence emission, ${ }^{29}$ owing to the exciton's generation demonstrated in Fig. 3f. To understand this $\mathrm{J} / \mathrm{H}$-aggregated exciton generation, which helps to boost the device efficiency, we compared the TRPL and TRANES analysis of 2 days and 1-month-old ampicillin as shown in Fig. 4a-o and Supplementary Fig. 2. In 2 days, fresh ampicillin-PEDOT:PSS mixtures, we observed a strong excimer emission in the region $500-750 \mathrm{~nm}$, in addition to the main fluorescence peak $(460 \mathrm{~nm})$, which confirmed the presence of Jaggregation (horizontal dipole interaction) in 25\% ampicillin-
PEDOT:PSS thin film (Fig. 4a, g) ${ }^{49-51}$ Interestingly, increased ampicillin concentration in PEDOT:PSS (i.e., at 40\%) improved the number of monomers more than it did in a $25 \%$ mixture, hence another type of interfacial dipole (inclined dipole interaction, $\mathrm{H}$ aggregation) was generated in $40 \%$ ampicillinPEDOT:PSS (shown in Fig. 2, Fig. 3b) by breaking the lactam ring of the previously attached ampicillin monomer, to form dimers. This is in accordance with the ampicillin molecular changes at high concentration and $\mathrm{pH}$, as reported before. ${ }^{55}$ Owing to the more perpendicular orientation, or stacking structure (Fig. 2) of these occasionally occurring dimers, we proposed the formation of H-type aggregation 51,56 in addition to J-aggregation for $40 \%$ ampicillin-PEDOT:PSS. TRANES analysis of $40 \%$ showed the excimer emission (Fig. 4h) with a slight blue-shift (at $530 \mathrm{~nm}$ peak) as compared with 25\% (550 nm peak) (Fig. $4 \mathrm{~g}$ ). This peak was attributed to the emission by allowed transition to the ground state from the lower-energy excited state ${ }^{56}$ of the occasional $\mathrm{H}$ aggregations as shown in Figs. $3 \mathrm{~b}, 4 \mathrm{~h}$. In addition to the TRANES 
analysis, we could conclude the formation of $\mathrm{J} / \mathrm{H}$ aggregation in ampicillin-PEDOT:PSS layers by analyzing the horizontal/ inclined molecular orientation using S-parameter (Supplementary Fig. 5). Interestingly, the above-mentioned additional emissions encountered around $500-750 \mathrm{~nm}$ in TRANES could not be realized in 1-month-old samples (Fig. 4e, k). This clearly conveyed the fact that after 1 month, $/ \mathrm{H}$-aggregated excitons were affected due to the generation of trimer and polymer in the mixtures as shown in Supplementary Fig. 6. In the case of $75 \%$ TRANES data, excimer emission shows a red-shift owing to the decomposed form of ampicillin as explained in Fig. 2. To eliminate the substrate-oriented emissions, we recorded TRANES for the same set of samples deposited on fused silica substrate (Fig. $4 \mathrm{p}-\mathrm{r}$ ), which reiterates the aggregations mentioned above (and ITO substrate peak as shown in Supplementary Fig. 2).

Moreover, we analyzed the solid-state PL spectra (Fig. 4s) for the pristine and ampicillin incorporated PEDOT:PSS layers where we could not obtain a proper emission by the pristine PEDOT: PSS. However, the PL spectra was obtained only when the ampicillin was added to the PEDOT:PSS, which indicated that the $\mathrm{J} / \mathrm{H}$-aggregated excitons would have contributed to the cultivation of more photons from the devices. Also, a shorter exciton lifetime (TRPL) was observed for $25 \%$ ampicillin-PEDOT:PSS (inset Fig. 4 s) as compared with $40 \%$. This is in accordance with the Jaggregations (horizontal dipole with shorter lifetime) and $\mathrm{H}$ aggregations (inclined dipole and longer lifetime) mechanism reported before. ${ }^{51,56}$ At $75 \%$, the new longer lifetimes can be attributed to the formation of new composites based on ampicillin and PEDOT:PSS, as shown in the inset of Fig. 4i. Two mechanisms could be invoked for the origin of the redshifted emission in 75\% ampicillin-PEDOT:PSS-(1) the aggregation between chromophores ${ }^{57}$ is dominant in this condition, and may induce fast excimer formation at an early stage, or (2) the aggregation between ampicillin and PEDOT:PSS is negligible. Here, the polymer matrix is further modified to reveal that each chromophore has more rigid and planar conformation in ground state. Some portion of chromophores, whose configuration is suitable for the intermolecular reaction, shows weak emission at $\sim 555 \mathrm{~nm}$ of $75 \%$. The other portion follows the conformational change observed in the 25 and $40 \%$ cases, and then shows the long decay lifetimes because of the rigid geometry from the aggregation.

At high concentration (75\%), ampicillin became unstable and it decomposed to conjugated forms such as penicilloic acid, penilloic acid, and 2-Hydroxy-3-phenylpyrazine. ${ }^{46-48}$ HPLC analysis (Supplementary Fig. 4) of 1-month-old 75\% ampicillinPEDOT:PSS confirmed this possible polymerization/conjugated forms. In addition, nuclear magnetic resonance (NMR) data presented in Supplementary Fig. 7 further reiterates the formation of polymer moieties. ${ }^{58}$ The interfacial dipole at $75 \%$ is speculated to be gone, as its presence has not been observed in such conjugated forms previously either. ${ }^{46-48}$ The highest alkaline nature of $75 \%$ sample is further tested, and confirmed with contact angle (CA) measurements (Supplementary Fig. 7), which expect to solve the acidic problems created by PEDOT:PSS on device electrodes. ${ }^{59}$

Performance enhancement by charge balance and J/H aggregations. The performance of single-unit OLED devices containing $0-75 \%$ of ampicillin-PEDOT:PSS is summarized in Fig. 5, Tables 1 and 2. Efficient light emission in an OLED is possible through charge (electron-hole) balance, by avoiding the recombination near the electrodes and confining within the EML. ${ }^{32,33,38}$ The device without ampicillin showed a low CE of $\sim 48 \mathrm{~cd} \mathrm{~A}^{-1}$. This is because the electron injection/mobility in $\mathrm{CBP}+\operatorname{Ir}(\mathrm{ppy})_{3}$ based EML through electron transport layer (ETL) is weaker than the hole injection/mobility from PEDOT:PSS. Therefore, more holes could reach the EML following the Ohmic-type contact, causing a large charge imbalance (Supplementary Fig. 8). With an addition of ampicillin in PEDOT:PSS in small concentrations (2, $10,15 \%)$ we analyzed a linear increase in device performance with increase in concentration of ampicillin as shown in Supplementary Fig. 9. However, a significant increase in OLED performance was observed by utilization of $25 \%$ ampicillin-PEDOT:PSS layer, with $\mathrm{CE}, \mathrm{EQE}$, and power efficiency (PE) values of $120 \mathrm{~cd} \mathrm{~A}^{-1}$, $35.1 \%$, and $70 \mathrm{lmW}^{-1}$, respectively, (Fig. $5 \mathrm{a}-\mathrm{d}$ ) in the green wavelength region $(495-570 \mathrm{~nm}$, electroluminance (EL) of the devices shown in Supplementary Fig. 9). As electron injection remained constant for all ampicillin-PEDOT:PSS devices, a suitable hole suppression was provided by $25 \%$ ampicillin-PEDOT: PSS layer observed by hole-only devices (HODs) as shown in Supplementary Fig. 8. The hole mobility calculation for ampicillin-PEDOT:PSS layers in relation to electron injection/mobility of the ETL (TPBi) layer were also analyzed at low- and highvoltage regions, which demonstrated the closest match for $25 \%$ ampicillin-PEDOT:PSS device. This hole suppression owing to an adequate decrease in $\varphi$ (caused by an opposite interfacial dipole towards anode) would be critically beneficial for the charge balance as shown in Supplementary Fig. 5, where the lowest injection difference $(0.11 \mathrm{eV})$ between holes and electrons was observed for 25\% ampicillin-PEDOT:PSS device.

When the holes injected from the ITO combined with the electrons in ampicillin-PEDOT:PSS layer, they formed excitons owing to the presence of an interfacial dipole. These $\mathrm{J} / \mathrm{H}$ aggregated extra or additional excitons (4 6\%) when dissociated resulted in the generation of photons at the ampicillin-PEDOT: PSS layer in addition to the emitted photons (25 30\%) from the EML layer. These excimer/exciton generation in ampicillinPEDOT:PSS layer was confirmed using TRANES and TRPL data in 500-600 nm wavelength region (Fig. 4). The contribution of these excitons in the device was analyzed using the device performance by heterojunction devices (Fig. 3e, without EML). This indicated that the addition of ampicillin (25\%) enhanced the efficiency by at least $4-6 \%$ in addition to the EML efficiency, thus delivering one of the top device performances. ${ }^{32,33,38}$ The variable-angle performance (shown as inset in Fig. 5c) demonstrated that including 25\% ampicillin-PEDOT:PSS device, all OLED devices (solid colored lines) presented near-perfect Lambertian emission compared with the simulated ideal (dotted line) curve. The quantum efficiency of the devices was crossexamined with half-moon integrating sphere measurement system (at $7 \pm 1 \mathrm{~V}$ using one-point measurement) (Fig. 5e) and was found to be similar with the obtained performance. To further understand the contribution of ampicillin's molecular structure, specific interfacial dipole and J-aggregations in the ampicillin-based OLEDs, we incorporated penicillin in PEDOT: PSS and fabricated the OLED devices (with the same layout) as shown in Fig. 5f. The major difference between ampicillin and penicillin is the absence of primary $-\mathrm{NH}_{2}$ group and can be attributed to the presence of weaker interfacial dipole than ampicillin. The comparison shows that the penicillin-PEDOT:PSS devices demonstrated an EQE of $\sim 24 \%$ in comparison with ampicillin-PEDOT:PSS device with an EQE of $\sim 35 \%$. This emphasizes that $\sim 11 \%$ EQE was additionally reinforced (boosted). As mentioned before this enhanced EQE as compared to the devices with penicillin could be due to charge balance and the formation of $\mathrm{J} / \mathrm{H}$-aggregated excitons. The injected holes from ITO and the electrons in the ampicillin-PEDOT:PSS layer combined to form excitons, which were dissociated causing an emission. The emission by these excitons in addition to that by the EML led to the overall increase of the resulting luminance. 
a

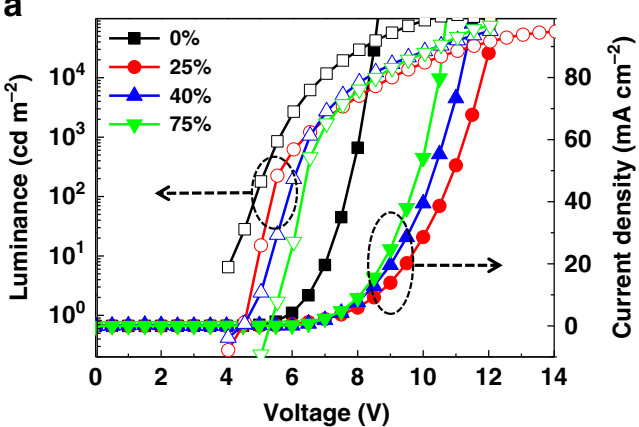

C

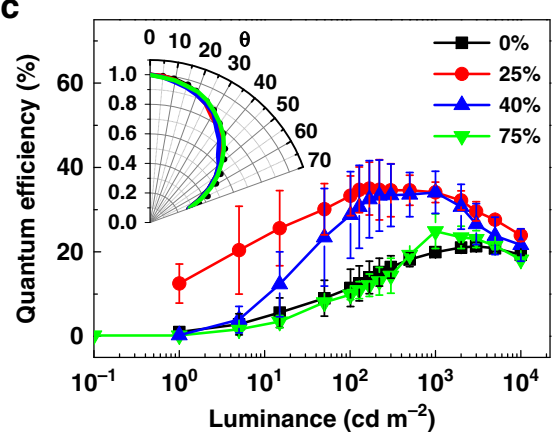

e

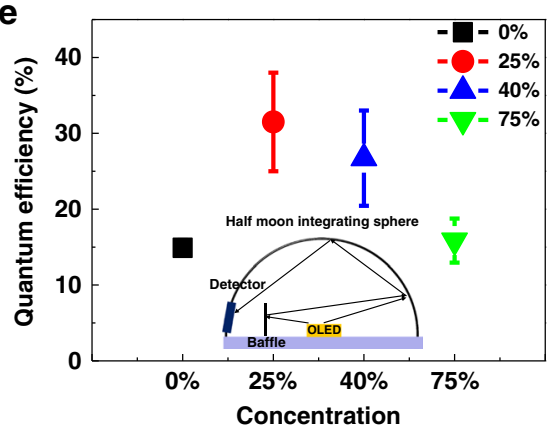

b

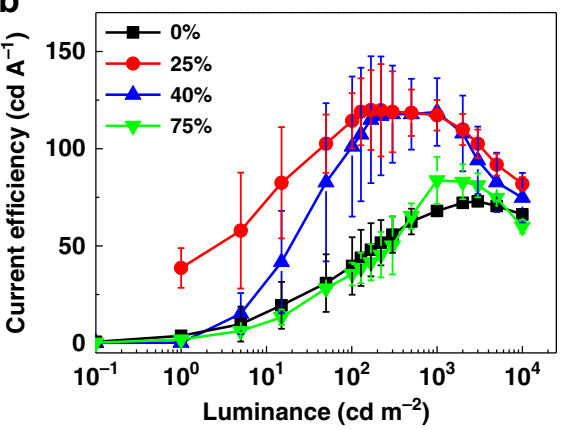

d

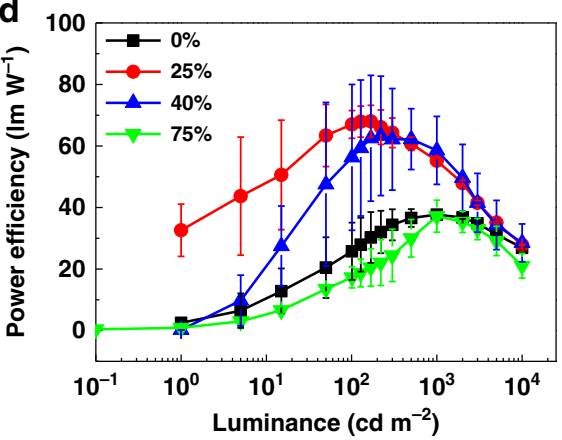

f

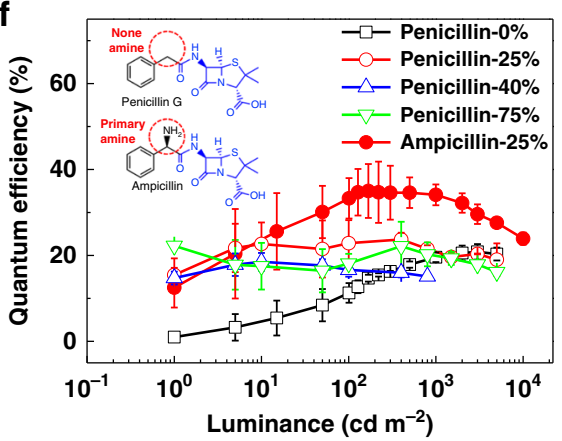

Fig. 5 Device performance analysis of organic light-emitting diodes (OLEDs) with (0-75\%) ampicillin-PEDOT:PSS. a J-V and luminance analysis showing the trend of current density. b Current efficiency (CE) analysis demonstrating 25\% ampicillin-PEDOT:PSS with best efficiency (of $120 \mathrm{~cd} \mathrm{~A}^{-1}$ ). $\mathbf{c}$ External quantum efficiency (EQE) of the devices. Inset showing Lambertian emission using the variable-angle analysis (luminance) obtained from the devices (solid lines) with the ideal Lambertian pattern (dotted line). d Power efficiency (PE) of the devices. e EQE measurement using half-moon integrating sphere. Inset showing schematic of the total luminance flux measurement system (half-moon integrating sphere) analyzed at ( $7 \pm 1 \mathrm{~V}$ ) using one-point-measurement. f EQE comparison with penicillin (without primary $-\mathrm{NH}_{2}$ ) based devices. Inset showing the difference of the chemical structure between penicillin (without primary $-\mathrm{NH}_{2}$ ) and ampicillin (with primary $-\mathrm{NH}_{2}$ ). Errors in $\mathbf{b}-\mathbf{f}$ are standard deviation from the mean, obtained averaging eight measurements

Table 1 Comparison of (0-75\%) ampicillin-PEDOT:PSS device performances current efficiency (CE), external quantum efficiency (EQE), and power efficiency (PE) with the standard deviations at the low luminance region $\left(1170 \mathrm{~cd} / \mathrm{m}^{2}\right)$ along with their color coordinates (CIE (International Commission on Illumination)-1931)

\begin{tabular}{|c|c|c|c|c|}
\hline Luminance $\left(\mathrm{cd} \mathrm{m}^{-2}\right)$ & Current efficiency $\left(\mathrm{cd} \mathrm{A}^{-1}\right)$ & Quantum efficiency (\%) & Power efficiency (Im W-1) & CIE color coordinates $(x, y)$ \\
\hline 1 & $3.8 \pm 0.42$ & $1 \pm 0.1$ & $2.5 \pm 0.3$ & $(0.296,0.625)$ \\
\hline 170 & $48.6 \pm 13.6$ & $14.1 \pm 3.8$ & $30.4 \pm 8.25$ & (at $4.5 \vee \& 143.6 \mathrm{~cd} \mathrm{~m}^{-2}$ ) \\
\hline 1 & $38.7 \pm 10.2$ & $12.5 \pm 4.6$ & $32.6 \pm 8.5$ & $(0.286,0.633)$ \\
\hline 168 & $120 \pm 20.3$ & $35.1 \pm 6.2$ & $68.1 \pm 5.3$ & (at $6 \vee \& 86.0 \mathrm{~cd} \mathrm{~m}^{-2}$ ) \\
\hline 1 & $0.25 \pm 0.03$ & $0.24 \pm 0.03$ & $0.21 \pm 0.02$ & $(0.285,0.630)$ \\
\hline 170 & $114.1 \pm 32.6$ & $32.4 \pm 9.1$ & $62.2 \pm 20.4$ & (at $5 \vee \& 2.5 \mathrm{~cd} \mathrm{~m}^{-2}$ ) \\
\hline 1 & $1.9 \pm 0.2$ & $0.2 \pm 0.09$ & $0.9 \pm 0.1$ & $(0.309,0.620)$ \\
\hline 170 & $42.7 \pm 9.3$ & $13.2 \pm 2.6$ & $20.4 \pm 6.1$ & (at $6.5 \mathrm{~V} \& 60.1 \mathrm{~cd} \mathrm{~m}^{-2}$ ) \\
\hline
\end{tabular}


Table 2 Comparison of (0-75\%) ampicillin-PEDOT:PSS device performances current efficiency (CE), external quantum efficiency (EQE), and power efficiency (PE) with the standard deviations at high luminance regions $\left(1000\right.$ and $\left.2000 \mathrm{~cd} / \mathrm{m}^{2}\right)$ along with their color coordinates (CIE (International Commission on Illumination)-1931)

\begin{tabular}{|c|c|c|c|c|c|}
\hline & Luminance $\left(\mathrm{cd} \mathrm{m}^{-2}\right)$ & Current efficiency $\left(c d A^{-1}\right)$ & Quantum efficiency (\%) & Power efficiency $\left(\operatorname{Im} W^{-1}\right)$ & CIE color coordinates $(x, y)$ \\
\hline $0 \%$ & 1000 & $67.7 \pm 1.9$ & $19.7 \pm 0.5$ & $37.7 \pm 1$ & $(0.294,0.617)$ \\
\hline $25 \%$ & 1000 & $117.6 \pm 8.2$ & $34.3 \pm 2.7$ & $55.2 \pm 0.2$ & $(0.286,0.622)$ \\
\hline \multirow[t]{2}{*}{$40 \%$} & 1000 & $118.5 \pm 18.2$ & $34.1 \pm 4.9$ & $58.6 \pm 11.1$ & $(0.284,0.623)$ \\
\hline & 2000 & $107.3 \pm 19.1$ & $30.7 \pm 5.4$ & $49.7 \pm 10.9$ & (at $10.5 \mathrm{~V} \& 3402 \mathrm{~cd} \mathrm{~m}^{-2}$ ) \\
\hline \multirow[t]{2}{*}{$75 \%$} & 1000 & $83.8 \pm 11.9$ & $24.9 \pm 4.1$ & $37.2 \pm 5.2$ & $(0.310,0.616)$ \\
\hline & 2000 & $83 \pm 9$ & $23.5 \pm 2.6$ & $35.3 \pm 3.5$ & (at $9.5 \vee \& 3338 \mathrm{~cd} \mathrm{~m}^{-2}$ ) \\
\hline
\end{tabular}

Interestingly, 40\% ampicillin-PEDOT:PSS infused devices also demonstrated comparable CE of $118 \mathrm{~cd} \mathrm{~A}^{-1}$, EQE of $33 \%$, and PE of $58.6 \mathrm{lmW}^{-1}$, especially in the higher luminance region. The larger decrease in $\varphi$ and hole injection by $40 \%$ ampicillinPEDOT:PSS layer would not be well-matched with the electron injection from the ETL, which could have induced a charge imbalance in EML at low luminance region. However, we speculate that at higher luminance (increased voltage) hole injection was improved (became similar to $25 \%$ ) owing to overcome of conduction barrier (Supplementary Figs. 8, 10), which could consequently lead to better charge balance thus improving the efficiency. Occasionally, a large fluctuation in $40 \%$ ampicillin-PEDOT:PSS devices efficiency was observed (shown as standard deviation in Fig. 5b-d) and on investigation the reason was found to be the absence/presence of long rod-like ampicillin crystals $^{60,61}$ (Supplementary Fig. 11) in the spin coated $40 \%$ ampicillin-PEDOT:PSS layer. As the ampicillin crystal formation could be supported by instability of various factors such as temperature ${ }^{60}$ and $\mathrm{pH}^{61}$, the temperature was kept constant during the preparation of ampicillin solution. However, as the $\mathrm{pH}$ of $40 \%$ ampicillin-PEDOT:PSS solution was around the same as reported crystal formation region $(\mathrm{pH} 7.8 \sim 6)^{61}$, the observed infrequent crystallization (Supplementary Fig. 11) could be owing to rare variations in $\mathrm{pH}$. We speculate that in the presence of such crystals the $40 \%$ ampicillin-PEDOT:PSS device efficiency fluctuated, but overall $25 \%$ and $40 \%$ ampicillin added devices showed remarkable improvement in device efficiency as compared with reference device (without ampicillin) (Tables 1,2).

In $75 \%$ concentrated ampicillin devices, the potential barrier for holes increased further, and hole injection was strongly suppressed, which caused a severe charge imbalance and reduced efficiency. The optimum conditions of ampicillin for the best OLED efficiency was found to be $50 \mathrm{mg} \mathrm{ml}^{-1}$ (ampicillin/sterilized deionized water (SDIW) having a maximum shelf-life of 2 days, kept at $2 \sim 5^{\circ} \mathrm{C}$ in a refrigerator. The role of ampicillin-PEDOT: PSS shelf-life and concentration $\left(\mathrm{mg} \mathrm{ml}^{-1}\right)$ of ampicillin-PEDOT: PSS were investigated using device fabrication (Supplementary Fig. 12) and devising the chemical interaction of 1-month-old ampicillin (Supplementary Fig. 6) for better understanding. Hence, we concluded that the $\mathrm{pH}$ control by ampicillinPEDOT:PSS preservation in refrigerator $\left(2-5^{\circ} \mathrm{C}\right)$ are the key factors to attain efficient performances in OLEDs. Figure $6 a-d$ demonstrate the schematic of the overall mechanism of the ampicillin-PEDOT:PSS layers with different concentrations. It has been configured based on the obtained charge injection, orientation of interfacial dipole, $\mathrm{J} / \mathrm{H}$ aggregations, chromophores interaction and the resulting contribution in the device efficiencies. The energy band diagram illustrated in Fig. $6 \mathrm{e}-\mathrm{h}$ exemplifies the vacuum level shift and hole/electron barrier from UPS, XPS, and HOD/electron-only devices investigations, with various ampicillin volume concentrations. Furthermore, the UPS analysis of the ampicillin-PEDOT:PSS with different thicknesses of the NPB layer $(1-20 \mathrm{~nm})$ was analyzed and the interface barriers/vacuum level shifts were investigated as shown in Supplementary Fig. 13. The ampicillin-PEDOT:PSS layer was also applied in forward and inverted OPVs as transport layers (data not shown), where inverted OPV with ampicillin-PEDOT: PSS exhibited superior performance than the forward structure, due to the decreased $\mathrm{E}_{f}$.

\section{Discussion}

In this work, we combined ampicillin with PEDOT:PSS to enhance the performance of OLEDs. With an increase in the concentration of ampicillin in PEDOT:PSS, a decrease in the $\varphi$ was analyzed. The presence of primary, secondary, and tertiary $-\mathrm{NH}_{2}$ along with the lactam ring in the ampicillin's molecular structure provided a specific charge distribution, which resulted in an interfacial dipole with high magnitude. With $25 \%$ ampicillin addition, a strong horizontal interfacial dipole, efficient Jaggregations and enhanced charge balance was achieved in the OLED devices. In particular, we measure a CE of $120 \mathrm{~cd} \mathrm{~A}^{-1}$, EQE of $35 \%$ and $\mathrm{PE}$ of $70 \mathrm{lmW}^{-1}$, which to our knowledge is among the highest reported performances for green ph-OLEDs. The achieved high-device efficiency was cross-evaluated with penicillin-based (without primary $-\mathrm{NH}_{2}$ ) devices. We observed that the penicillin-based devices showed an EQE of 24\%, which was $\sim 11 \%$ less than the ampicillin-based devices (35.1\%). Hence, it could be concluded that the enhanced performance by ampicillin-PEDOT:PSS device was due to the presence of horizontal interfacial dipole and exciton emission from J-aggregations. Comparable device efficiencies were observed for $40 \%$ ampicillinPEDOT:PSS devices as well, however, the ampicillin's crystallization caused strong deviations in the obtained results. On the other hand, 25\% ampicillin-PEDOT:PSS devices showed a quite stable performance, which makes this concentration preferable for OLED applications. A wide range of properties obtained by the combination of ampicillin in PEDOT: PSS represents a significant advance towards the fabrication of highly efficient biocompatible AIs, optoelectronic, and biomedical devices.

\section{Methods}

ITO glass substrate cleaning and surface treatment. The patterned ITO glass substrate was cleaned by acetone and isopropyl alcohol (IPA) for $10 \mathrm{~min}$ using sonication at $40 \mathrm{kHz}$. ITO substrate was boiled in IPA for $15 \mathrm{~min}$ and finally treated with ultraviolet-ozone $(254 \mathrm{~nm})$ for $20 \mathrm{~min}$. The sheet resistance of ITO substrate was $15 \Omega \mathrm{sq}^{-1}$.

Ampicillin preparation with PEDOT:PSS. Ampicillin aqueous solution was prepared by dissolving $50 \mathrm{mg}$ ampicillin powder (Purchased from VWR AMRESCO 99.0\% purity, USP Grade) in $1 \mathrm{ml}$ of SDIW. The 1-D (one-time distilled) SDIW was prepared by sterilizing the DIW in an autoclave equipment at $121^{\circ} \mathrm{C}$ for 15 mins. The SDIW was with a neutral $\mathrm{pH}(\sim 7.5)$ and a resistance of $\sim 0.18 \mathrm{M} \Omega \mathrm{cm}$. Ampicillin dissolved SDIW solution was filtered using $21 \mu \mathrm{m}$ size meshed filter, 
a

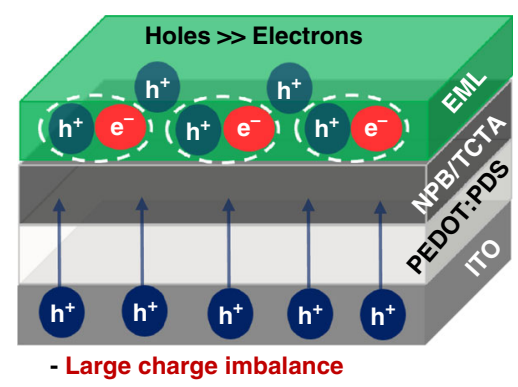

c

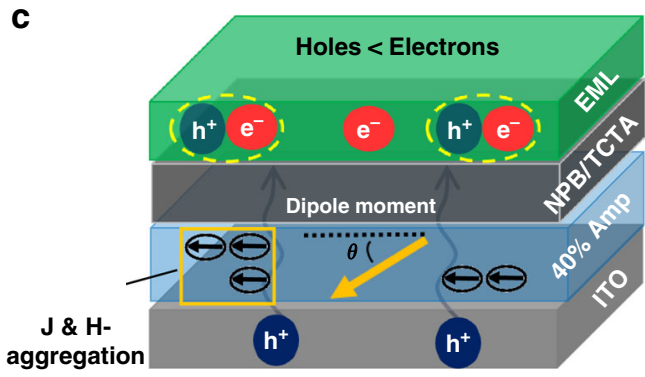

- Slight charge imbalance b

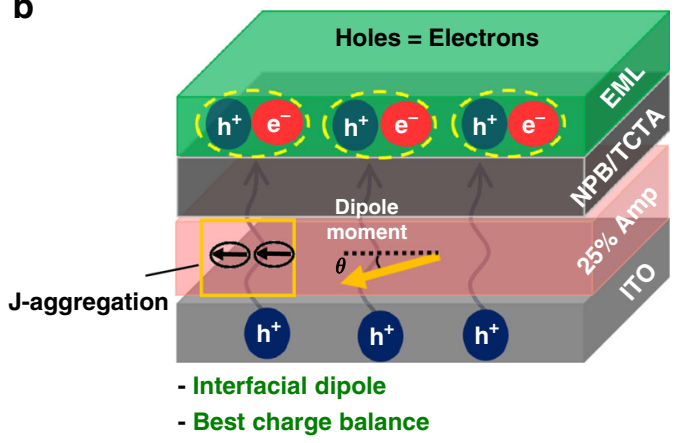

d

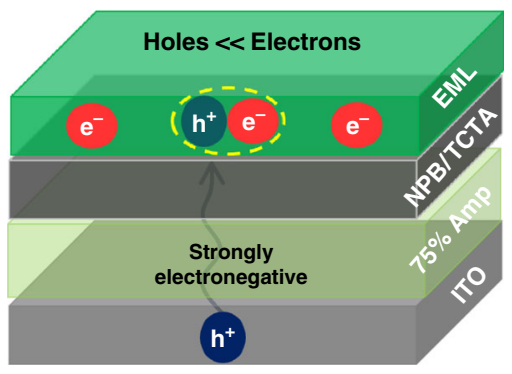

- Large charge imbalance e

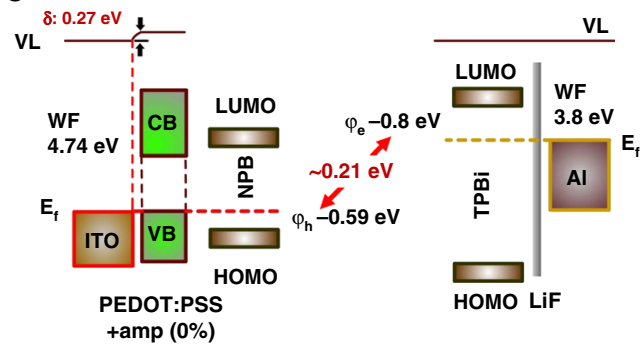

g

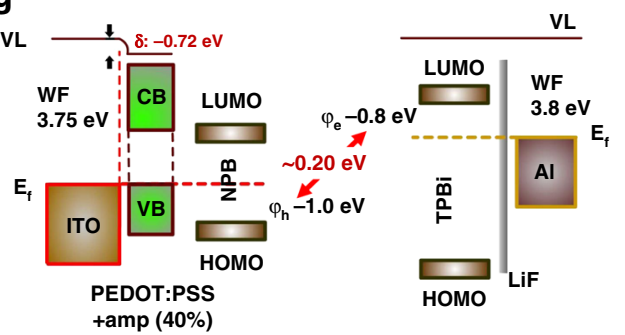

f

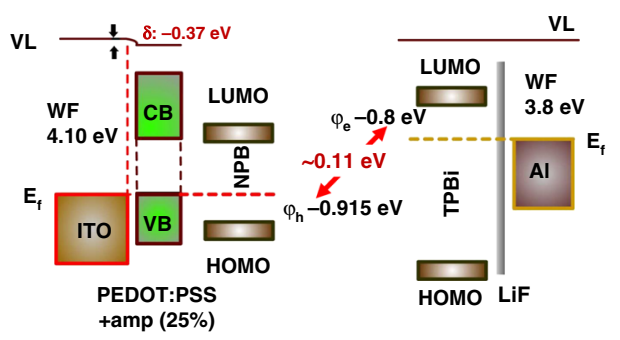

h

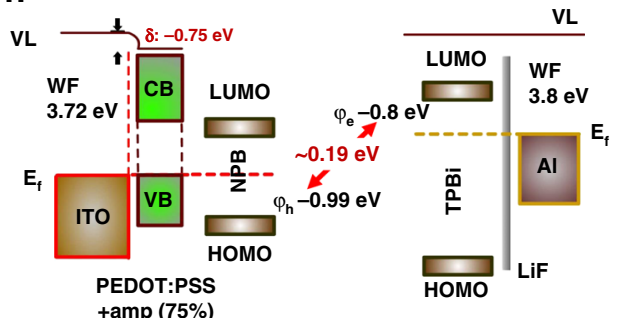

Fig. 6 Schematic of the overall mechanism and role of (0-75\%) ampicillin-PEDOT:PSS layers with Fermi levels ( $E_{f}$ ) alignment for a PEDOT:PSS only device, b $25 \%$ ampicillin-PEDOT:PSS. The interfacial dipole drawn as a yellow arrow directed towards anode. The hole injection and electron injection are shown as blue and red spheres, respectively. The dotted circles around hole-electron pair show the charge recombination. c 40\% ampicillin-PEDOT:PSS. d 75\% ampicillin-PEDOT:PSS with similar information. e-h Energy-level alignment with 0-75\% ampicillin-PEDOT:PSS concentrations illustrated from hole-onlydevice (HOD) measurement. Vacuum level shift $(\delta$ ) mentioned by ultra-violet photoelectron spectroscopy (UPS) analysis. The red arrows demonstrate the difference between the hole and electron injection barriers

which could screen out unwanted biomaterials with even very small sizes (i.e., < $40 \mu \mathrm{m})$. The filter was also utilized to remove the ampicillin crystals formed by variations in the $\mathrm{pH}$ of the solution. Different concentrations $(25 \%, 40 \%$, and $75 \%$ with volume ratios of $1 \mathrm{ml}: 3 \mathrm{ml}, 2 \mathrm{ml}: 3 \mathrm{ml}$, and $3 \mathrm{ml}: 1 \mathrm{ml}$, respectively) of ampicillin solution was added to PEDOT:PSS (CLEVIOS P VP AL 4083, Heraeus). Devices with lower concentrations (2-15\%) of ampicillin in PEDOT:PSS were also fabricated using the same forward device structure for comparison purposes. The amounts and ratios of all concentrations from 2 to $75 \%$ of ampicillin in PEDOT: PSS have been presented in Supplementary Fig. 13. Further, during the preparation of ampicillin-PEDOT:PSS, 25-40\% solutions exuded an ammonia-like smell, whereas $75 \%$ solution did not exhibit any kind of smell. This indicated the extent of evaporation or removal of $\mathrm{CO}_{2}$ gas molecules in such solutions. Moreover, the device performances are quite dependent on the $\mathrm{pH}$ of the ampicillin-PEDOT: PSS solution status and should be properly monitored for reproducibility of the results. The penicillin solution was also prepared using the same method mentioned above.

OLED device fabrication. In all, (0-75\%) ampicillin-PEDOT:PSS as a hole injection layer was spin coated onto the cleaned ITO glass with a spin speed of $4000 \mathrm{rpm}$ for $30 \mathrm{~s}$ and baked at $120^{\circ} \mathrm{C}$ for $10 \mathrm{~min}$. NPB as HTL $(\sim 20 \mathrm{~nm})$ was deposited by thermal evaporation with a base pressure of $5.0 \times 10^{-8}$ torr. TCTA as an exciton blocking layer with thickness $10 \mathrm{~nm}$ was deposited on NPB. CBP and Ir $(\mathrm{ppy})_{3}(15 \mathrm{~nm}, 8 \%$ doping) were combined as a thin EML $(15 \mathrm{~nm})$. This EML has been reported to have a random orientation, ${ }^{62}$ therefore does not have a critical effect in our work. TPBi $(20 \mathrm{~nm})$ was deposited as an ETL layer and the effect of ETL thickness on the charge balance has been investigated before. ${ }^{63}$ The thin EML usually requires a thin ETL for the optimized charge balance or better device 
performance, and vice versa. It was observed that low thicknesses $(<50 \mathrm{~nm})$ were preferable for better device performance owing to the exciton confinement while the high thickness $(>50 \mathrm{~nm}$ ) resulted in an efficiency roll-off. LiF/Al cathode was patterned on ETL using shadow masks $\left(4 \mathrm{~mm}^{2}\right)$ by thermal evaporation with a base pressure of $5.0 \times 10^{-8}$ torr. The deposition rates were fixed to $0.2 \AA \mathrm{s}^{-1}$ and $8 \AA \mathrm{s}^{-1}$ for the $\mathrm{LiF}$ and $\mathrm{Al}$ electrodes. $\mathrm{LiF}$ and $\mathrm{Al}$ thicknesses were $1 \mathrm{~nm}$ and $120 \mathrm{~nm}$, respectively, which were analyzed using crystal quartz and re-confirmed using the surface profiler. The tooling process was introduced in Supplementary Fig. 14, and three kinds of different evaporators were utilized to confirm the reproducibility of the device performance. The penicillin-based OLED devices were also prepared using the same method mentioned above.

XPS/UPS analysis. XPS (AXIS-NOVA, Kratos. Inc.) with monochromatic Al-Ka $(1486.6 \mathrm{eV})$ radiation as a photon source with a hemispherical analyzer was employed to investigate the bonding of the species $(C-1 s, N-1 s$, and $S-2 p)$ in the sample (base pressure $=1 \times 10^{-9}$ torr). The resolution of the electron analyzer was $0.47 \mathrm{eV}$ as measured from the full width at half maximum (FWHM) of the $\mathrm{Ag} 3 \mathrm{~d}_{5 / 2}$ peak. The binding energy of XPS spectra was calibrated by the position of C-1s $(285.0 \mathrm{eV})$ of adventitious carbons in the air. High-resolution XPS spectra were obtained using an analysis area of $400 \mu \mathrm{m}$ of the $40 \mathrm{eV}$ pass energy with an energy step of $0.05 \mathrm{eV}$. UPS with an excitation energy of He I $(21.2 \mathrm{eV})$, which is integrated with XPS, was performed to characterize the electronic structure of the PEDOT: PSS. The UPS spectra were obtained using the sample biased at $-15.0 \mathrm{~V}$ to clear the secondary cutoff. An energy resolution in UPS of $0.05 \mathrm{eV}$ was determined from the slope of the Fermi edge of a cleaned polycrystalline Au surface.

AFM analysis. The surface morphology of ampicillin-PEDOT:PSS layers was analyzed with a multimode 8 AFM (Bruker) equipped with a Nanoscope V controller. We used Al coated silicon probe (NCHR, Nanoworld, Germany) with a nominal spring constant of $40 \mathrm{Nm}^{-1}$ and a resonance frequency of $320 \mathrm{kHz}$. All images were obtained in tapping mode AFM at room temperature, with $384 \times 384$ pixels in a $3 \times 3 \mu \mathrm{m}$ of scan size at a scan rate of $1 \mathrm{~Hz}$.

XRD analysis. The structural characteristics of the samples were measured using a PANalytical X'Pert PRO-MPD XRD equipped with X-ray mirrors (as an incident and a diffracted optics), and operated with $\mathrm{Cu}-\mathrm{Ka}$ radiation at $40 \mathrm{kV}$ and $30 \mathrm{~mA}$. The crystallite size of the samples was determined by means of XRD, using the Scherrer equation $\left(L=\frac{K \lambda}{\beta \cos \theta}\right)$.

HR-TEM analysis. Structural properties of 25\% ampicillin-PEDOT:PSS layers were analyzed using HR-TEM with JEOL-ARM $200 \mathrm{~F}$ (JEOL, USA Inc,) microscope operating at $200 \mathrm{kV}$ with a point-to-point resolution of $0.19 \mathrm{~nm}$.

Optical Properties (transmittance and absorption analysis). UV-vis spectrophotometer (JASCO V-570) was utilized for light transmittance and absorbance spectra.

HPLC and NMR analysis. The samples were analyzed by reverse-phase HPLC conducted under the following conditions: the reaction samples were applied to a Mightysil RP-18 GP column $(4.6 \times 250 \mathrm{~mm}, 5 \mu \mathrm{m}$; KANTO Reagents, Japan), elution of ampicillin-PEDOT:PSS (0-20 min, 75-0\% B and 20-25 $\mathrm{min}, 0-75 \% \mathrm{~B}$; where $\mathrm{A}=$ water containing $0.1 \%$ trifluoroacetic acid and $\mathrm{B}=$ Acetonitrile) was monitored at $259 \mathrm{~nm}$, at a flow rate of $0.5 \mathrm{~mL} \mathrm{~min}^{-1}$ at $30^{\circ} \mathrm{C}$. HPLC analysis was beneficial for analyzing molecules with low molecular weight such as ampicillin. Even though, gas-pressure chromatography) ${ }^{64}$ would have been beneficial to characterize large molecule weight polymers such as PEDOT:PSS but, we could observe the -PSS peak by HPLC and a minor shift in retention time as well by the addition of ampicillin.

For proton $\left({ }^{1} \mathrm{H}\right) \mathrm{NMR}$, crude samples of $75 \%$ ampicillin-PEDOT:PSS were dried by lyophilization and dissolved in dimethyl sulfoxide-d6 followed by centrifugation at $12,000 \mathrm{rpm}$ to remove the insoluble components. NMR was performed to clarify the structures of both samples. NMR was performed using Varian Unity Inova $300 \mathrm{MHz}$ spectrometry (Varian, USA) with a TCI CryoProbe $(5 \mathrm{~mm})$ and the data were analyzed by TopSpin 3.1 (Bruker) and MestReNova 8.0 (Mestrelab Research S. L., Spain) software.

TRPL/TRANES analysis. Picosecond TRPL/TRANES measurements were conducted using commercial fluorescence lifetime measurement system (Hamamatsu streak camera, C11200). A commercial optical parametric amplifier (TOPASprime, Light-Conversion) seeded with a commercial regenerative amplifier system (Spitfire-Ace, Spectra-Physics) operating at $1 \mathrm{KHz}$ was utilized as the light source. FWHM of the instrumental response function were $\sim 20$ ps in a 1 ns time window. All data were obtained in single photon counting mode using the Hamamatsu U8167 software.

Ellipsometry with refractive index and S-factor analysis. The refractive index and extinction coefficient for S-factor were investigated using spectroscopic ellipsometry (SE) of V-VASE (J.A. Woollam) with rotating analyzer system, in the wavelength range from 410 to $700 \mathrm{~nm}$, and incident angles of $65^{\circ}, 70^{\circ}$, and $75^{\circ}$. To calculate the S-factor, we performed anisotropic optical modeling (uniaxial model) in which the $x-y$ plane and the $z$ axis were considered to have different dielectric function properties. In addition, the optical model and the best-fit parameter values were calculated using the WVASE32 software (J.A. Woollam). Finally, based on the results of SE analysis, we calculated the molecular orientation parameter S using the following equation:

$$
S=\frac{3}{2}\left\langle\cos ^{2} \theta\right\rangle-\frac{1}{2}=\frac{k_{e}-k_{o}}{k_{e}+2 k_{o}}
$$

OLED device performance analysis. The J-V was analyzed using the probe station and Electroluminescence (EL) measurements were conducted using a Keithley 2400 voltmeter and Minolta CS-2000 spectrometer. For viewing angle measurement, the device performance (Luminance) was obtained by automatic rotating the device holding jig (stage) at various angles $0-70^{\circ}$ with a regular interval of $5^{\circ}$ (Supplementary Fig. 15). To avoid the OLED degradation after consecutive measurements from 0 to $70^{\circ}$ and 70 to $0^{\circ}$ (at each $5^{\circ}$ angle variation), average values were taken for analysis and multiplied with $\cos \theta$ in accordance to Lambertian cosine law $\left(I=\mathrm{I}_{0} \cdot \cos \theta\right)^{65}$. The obtained luminance from devices (solid lines) was plotted against the ideal Lambertian curve, which indicated that a nearperfect Lambertian (uniform) luminance.

Integrating sphere analysis. The EQE of the devices were cross-analyzed using integrating sphere, total luminance flux measurement system (Otsuka half-moon (HM) series) with constant voltage one-point measurement, (at $7 \pm 1 \mathrm{~V}$ ). The EL of OLEDs was measured at room temperature in a controlled voltage $(\mathrm{C}-\mathrm{V})$ mode and integration time of $5 \mathrm{~s}$. The spectrometer (LE-5400) CCD type, TE-cooled $(350-930 \mathrm{~nm})$ with 6 " hemi sphere having coating reflectance of $98 \%$ (spectraflect) were utilized. (Supplementary Fig. 15).

Conductivity measurement. A thick layer $(\sim 1 \mu \mathrm{m})$ of ampicillin-PEDOT:PSS was prepared on a glass substrates using the drop casting method. The conductivity was analyzed at a constant current of $100 \mathrm{nA}$ using 4-probe source measurement units (Keithly 236 source, 700 switch system, 6485 picoammeter by Jeong Yeon Systems, Korea).

Optical microscope. The surface morphology of the spin coated samples of ampicillin-PEDOT:PSS were analyzed using optical microscope (KSM-BA3T, Samwon scientific Ind. Co. Ltd., Korea)

Device lifetime measurement. The devices were encapsulated using a glass or acrylic adhesive tape before measurement. The analysis was conducted using an OLED lifetime test system (M6000, McScience Inc., Korea) enclosed in a chamber with controlled temperature and humidity. The tool provides multi-channel testing frame with various control mode power driving. The samples were analyzed at $1000 \mathrm{~cd} \mathrm{~m}^{-2}$ until $50 \%$ drop in device performance was observed.

CA analysis. The CA was analyzed using the smart drop standard (FEMTOBIOMED, South Korea) and this system uses the GEM 'single non-approximated algorithm numerical calculation of Bash forth-adams equation.

pH value measurement. The $\mathrm{pH}$ value was analyzed using the MP511 of $\mathrm{pH}$ meter (SANXIN, China) and $201 \mathrm{~T} \mathrm{pH}$ measuring electrode with an automatic temperature compensation function for a three-in-one electrode.

\section{Data availability}

The data that support the findings of this study are available from the corresponding author upon reasonable request.

Received: 30 January 2019; Accepted: 16 September 2019; Published online: 21 October 2019

\section{References}

1. Abbott, A. et al. 2016 in news: The science events that shaped the year. Nature 540, 496-499 (2016).

2. Wang, F.-Y. et al. Where does AlphaGo go: from Church-Turing thesis to AlphaGo thesis and beyond. IEEE/CAA J. Automat. Sin. 3, 113-120 (2016).

3. Waldrop, M. M. More than moore. Nature 530, 144 (2016).

4. Zafar, M., Kumar, S., Kumar, S. \& Dhiman, A. K. Artificial intelligence based modeling and optimization of poly (3-hydroxybutyrate-co-3-hydroxyvalerate) production process by using Azohydromonas lata MTCC 2311 from cane 
molasses supplemented with volatile fatty acids: a genetic algorithm paradigm. Bioresour Technol 104, 631-641 (2012).

5. Zang, Y., Zhang, F., Di, C.-a \& Zhu, D. Advances of flexible pressure sensors toward artificial intelligence and health care applications. Materials Horizons 2, 140-156 (2015).

6. Lim, F. J., Ananthanarayanan, K., Luther, J. \& Ho, G. W. Influence of a novel fluorosurfactant modified PEDOT: PSS hole transport layer on the performance of inverted organic solar cells. J. Mate. Chem. 22, 25057-25064 (2012).

7. De Kok, M. et al. Modification of PEDOT: PSS as hole injection layer in polymer LEDs. Phys. Status Solidi (a) 201, 1342-1359 (2004).

8. Zhang, W. et al. High-efficiency ITO-free polymer solar cells using highly conductive PEDOT: PSS/surfactant bilayer transparent anodes. Energy Environ. Sci. 6, 1956-1964 (2013).

9. Shi, H., Liu, C., Jiang, Q. \& Xu, J. Effective approaches to improve the electrical conductivity of PEDOT: PSS: a review. Adv. Electron. Mater. 1, 1500017 (2015).

10. Zhang, F., Johansson, M., Andersson, M. R., Hummelen, J. C. \& Inganäs, O. Polymer photovoltaic cells with conducting polymer anodes. Adv. Mater. 14, 662-665 (2002).

11. Ouyang, J., Chu, C. W., Chen, F. C., Xu, Q. \& Yang, Y. Polymer optoelectronic devices with high-conductivity poly (3, 4-ethylenedioxythiophene) anodes. J. Macromol. Sci. 41, 1497-1511 (2004).

12. Rivnay, J. et al. Structural control of mixed ionic and electronic transport in conducting polymers. Nat. Commun. 7, 11287 (2016).

13. Wang, C. \& Weiss, E. A. Accelerating FRET between near-infrared emitting quantum dots using a molecular $\mathrm{j}$-aggregate as an exciton bridge. Nano Lett. 17, 5666-5671 (2017).

14. Rolinson, G. \& Geddes, A. The 50th anniversary of the discovery of 6aminopenicillanic acid (6-APA). Int. J. Antimicrob. Agents 29, 3-8 (2007).

15. Acred, P., Brown, D., Turner, D. \& Wilson, M. Pharmacology and chemotherapy of ampicillin-a new broad-spectrum penicillin. $\mathrm{Br}$. J. Pharmacol. Hemother. 18, 356-369 (1962).

16. Akova, M. Sulbactam-containing $\beta$-lactamase inhibitor combinations. Clin. Microbiol. Infect. 14, 185-188 (2008).

17. Uehara, T., Dinh, T. \& Bernhardt, T. G. LytM-domain factors are required for daughter cell separation and rapid ampicillin-induced lysis in Escherichia coli. J. Acteriol. 191, 5094-5107 (2009).

18. Lin, Z. et al. A work-function tunable polyelectrolyte complex (PEI: PSS) as a cathode interfacial layer for inverted organic solar cells. J. Mater. Chem. A 2, 7788-7794 (2014).

19. Kang, H., Hong, S., Lee, J. \& Lee, K. Electrostatically self-assembled nonconjugated polyelectrolytes as an ideal interfacial layer for inverted polymer solar cells. Adv. Mater. 24, 3005-3009 (2012).

20. Tsang, S., Drolet, N., Tse, S., Tao, Y. \& Lu, Z. Impact of interfacial dipole on carrier transport in bulk heterojunction poly (3-hexylthiophene) and [6, 6]-phenyl C 61-butyric acid methyl ester blends. Appl. Phys. Lett. 97, 226 (2010).

21. Malloci, G., Serra, G., Bosin, A. \& Vargiu, A. V. Extracting conformational ensembles of small molecules from molecular dynamics simulations: ampicillin as a test case. Computation 4, 5 (2016).

22. Anzline, C., Israel, S., Devi, R. N., Sheeba, R. \& Rajkumar, P. R. High resolution synchrotron diffraction study on charge density distribution of ampicillin trihydrate. Chin. J. Chem. Phys. 30, 50-62 (2017).

23. Wu, F.-C., Tung, K.-C., Chou, W.-Y., Tang, F.-C. \& Cheng, H.-L. Charge selectivity in polymer: fullerene-based organic solar cells with a chemically linked polyethylenimine interlayer. Org. Electron. 29, 120-126 (2016).

24. Liang, Y., Wang, C., Li, J., Wang, L. \& Fu, J. The penicillin derivatives as corrosion inhibitors for mild steel in hydrochloric acid solution: experimental and theoretical studies. Int. J. Electrochem. Sci. 10, 8072-8086 (2015).

25. Deng, X. et al. Ultra-low work function transparent electrodes achieved by naturally occurring biomaterials for organic optoelectronic devices. $A d v$. Mater. Interfaces 1, 1400215 (2014).

26. Li, A. et al. Highly efficient inverted organic solar cells using amino acid modified indium tin oxide as cathode. Appl. Phys. Lett. 104, 49-51 (2014).

27. Nie, R. et al. Highly sensitive and broadband organic photodetectors with fast speed gain and large linear dynamic range at low forward bias. Small 13, 1603260 (2017).

28. Nie, R., Li, A. \& Deng, X. Environmentally friendly biomaterials as an interfacial layer for highly efficient and air-stable inverted organic solar cells. J. Mater. Chem. A 2, 6734-6739 (2014).

29. Kotagiri, N., Sakon, J., Han, H., Zharov, V. P. \& Kim, J.-W. Fluorescent ampicillin analogues as multifunctional disguising agents against opsonization. Nanoscale 8, 12658-12667 (2016).

30. Engelhard, S. \& Faisal, F. Quantum mechanical study of time-dependent energy transfer between perturbers in a Scheibe aggregate. J Chem. Phys. 110, 3596-3605 (1999).
31. Khan, T. M. et al. Organic photovoltaic cells with stable top metal electrodes modified with polyethylenimine. ACS Appl. Mater. Interfaces 6, 6202-6207 (2014).

32. Huang, C. et al. Thermally activated delayed fluorescence-based tandem OLEDs with very high external quantum efficiency. Phys. Status Solidi (a) 214 1700240 (2017).

33. $\mathrm{Xu}, \mathrm{T}$. et al. Highly simplified tandem organic light-emitting devices incorporating a green phosphorescence ultrathin emitter within a novel interface exciplex for high efficiency. ACS Appl. Mater. Interfaces $\mathbf{9}$, 10955-10962 (2017).

34. Komino, T. et al. Electroluminescence from completely horizontally oriented dye molecules. Appl. Phys. Lett. 108, 241106 (2016).

35. Moon, C. K. et al. Combined inter-and intramolecular charge-transfer processes for highly efficient fluorescent organic light-emitting diodes with reduced triplet exciton quenching. Adv. Mater. 29, 1606448 (2017).

36. Wu, T.-L. et al. Diboron compound-based organic light-emitting diodes with high efficiency and reduced efficiency roll-off. Nat. Photonics 12, 235 (2018).

37. Helander, M. et al. Chlorinated indium tin oxide electrodes with high work function for organic device compatibility. Science 332, 944-947 (2011).

38. Kim, S. Y. et al. Organic Light-Emitting diodes with $30 \%$ external quantum efficiency based on a horizontally oriented emitter. Advanced Funct. Mater. 23, 3896-3900 (2013).

39. Park, Y., So, Y., Chung, S. \& Jin, J. Photoelectron spectroscopy study of the electronic structures of poly (p-phenylenevinylene) derivatives. J. Korean Phys. Soc. 37, 59-63 (2000).

40. Peres, L., Bou, A., Cornille, C., Barakel, D. \& Torchio, P. Work function measurement of multilayer electrodes using Kelvin probe force microscopy. J. Phys. D Appl. Phys. 50, 13LT01 (2017).

41. Hwang, J., Amy, F. \& Kahn, A. Spectroscopic study on sputtered PEDOT. PSS: role of surface PSS layer. Org. Electron. 7, 387-396 (2006).

42. Yun, D.-J. et al. Characterizing annealing effect of poly $(3,4-$ ethylenedioxythiophene) polymerized with poly (4-styrenesulfonate) conjugated film on the molecular arrangement and work function by corelevel and valence-level band spectra. ECS J. Solid State Sci.Technol. 1, M10-M14 (2012).

43. Huang, P. et al. Catechol derivatives as dopants in PEDOT: PSS to improve the performance of $\mathrm{p}-\mathrm{i}-\mathrm{n}$ perovskite solar cells. J. Mater. Chem. 5, 24275-24281 (2017).

44. Park, H. et al. Enhanced thermoelectric properties of PEDOT: PSS nanofilms by a chemical dedoping process. J. Mater. Chem. 2, 6532-6539 (2014).

45. Jesuraj, P. J., Jeganathan, K., Navaneethan, M. \& Hayakawa, Y. Far-field and hole injection enhancement by noble metal nanoparticles in organic light emitting devices. Synth. Met. 211, 155-160 (2016).

46. Kessler, D. P., Cushman, M., Ghebre-Sellassie, I., Knevel, A. M. \& Hem, S. L. Investigation of a proposed penicillin $\mathrm{G}$ acidic degradation scheme using highpressure liquid chromatography and optimization techniques and mechanistic considerations. J. Chem.Soc. Perkin Trans. 2, 1699-1703 (1983).

47. Robinson-Fuentes, V., Jefferies, T. \& Branch, S. Degradation pathways of ampicillin in alkaline solutions. J. Pharm Pharmacol. 49, 843-851 (1997).

48. Masada, M., Kuroda, Y. \& Nakagawa, T. \& UNO, T. Structural investigation of new metabolites of aminopenicillins excreted in human urine. Chem. Pharm. Bull. 28, 3527-3536 (1980).

49. Mishra, R. K., Pulidindi, I. N., Kabha, E. \& Gedanken, A. In situ formation of carbon dots aids ampicillin sensing. Anal. Methods 8, 2441-2447 (2016).

50. Liu, M. \& Kira, A. Fabrication of J aggregate films in synthetic polyanion matrix and their chemochromism. Thin Solid Films 359, 104-107 (2000).

51. Pucci, A. \& Ruggeri, G. Mechanochromic polymer blends. J. Mater. Chem. 21, 8282-8291 (2011).

52. Peyratout, C. \& Daehne, L. Aggregation of thiacyanine derivatives on polyelectrolytes. Phys. Chem. Chem. Phys. 4, 3032-3039 (2002).

53. Brixner, T., Hildner, R., Köhler, J., Lambert, C. \& Würthner, F. Exciton transport in molecular aggregates-from natural antennas to synthetic chromophore systems. Adv. Energy Mater. 7, 1700236 (2017).

54. Qin, W. et al. Red emissive AIE luminogens with high hole-transporting properties for efficient non-doped OLEDs. Chem. Commun. 51, 7321-7324 (2015).

55. Bundgaard, H. \& Larsen, C. Polymerization of penicillins: IV. Separation, isolation and characterization of ampicillin polymers formed in aqueous solution. J. Chromatogr. 132, 51-59 (1977).

56. Chaudhuri, D. et al. Enhancing long-range exciton guiding in molecular nanowires by $\mathrm{H}$-aggregation lifetime engineering. Nano Lett. 11, 488-492 (2010).

57. Waluk, J. Conformational analysis of molecules in excited states. Vol. 22 (John Wiley \& Sons, USA, 2000).

58. Pek, G. Y., Bystrov, V., Kleiner, E., Blinova, I. \& Khokhlov, A. NMR spectroscopy of $\mathrm{H} 1$ of penicillins and their derivatives. Russ. Chem. Bull. 17, 2096-2103 (1968). 
59. Noh, Y.-J. et al. Efficient PEDOT: PSS-free polymer solar cells with an easily accessible polyacrylonitrile polymer material as a novel solution-processable anode interfacial layer. ACS App. Mater. Interfaces 7, 25032-25038 (2015).

60. Flórez-Acosta, O., Tobón-Zapata, G. \& Valencia-Velasquez, J. Categorization of the main descriptors of different ampicillin crystal habits. Braz. J. Pharm. Sci. 46, 679-685 (2010).

61. Encarnación-Gómez, L. G., Bommarius, A. S. \& Rousseau, R. W. Crystallization kinetics of ampicillin using online monitoring tools and robust parameter estimation. Ind. Eng. Chem. Res. 55, 2153-2162 (2016).

62. Kim, K. H., Moon, C. K., Lee, J. H., Kim, S. Y. \& Kim, J. J. Highly efficient organic light-emitting diodes with phosphorescent emitters having high quantum yield and horizontal orientation of transition dipole moments. Adv. Mater. 26, 3844-3847 (2014).

63. Lee, W. H. et al. Improvement of charge balance, recombination zone confinement, and low efficiency roll-off in green phosphorescent OLEDs by altering electron transport layer thickness. Mater. Res. Express 5, 076201 (2018).

64. Podzimek, S. \& Hyrsl, J. GPC and HPLC characterization of unsaturated polyester resins. J. Appl. Polym. Sci. 53, 1351-1356 (1994).

65. Kumar, A., Srivastava, R., Kamalasanan, M. \& Mehta, D. S. Enhancement of light extraction efficiency of organic light emitting diodes using nanostructured indium tin oxide. Opt. Lett. 37, 575-577 (2012).

\section{Acknowledgements}

We acknowledge support from the Basic Science Research Program through the National Research Foundation of Korea (NRF) funded by the Ministry of Education (NRF2017R1A2B4005583).

\section{Author contributions}

The manuscript was prepared through contributions from all authors. S.Y.R. is a corresponding author designed and conceived this work. H.H., D.H.K., and P.J.J. equally contributed in this work. H.H. and P.J.J. have consolidated the results and drafted the manuscript. D.H.K. and J.C.L. conducted all the experimental work. J.Y.S., S.H.R., W.H. L., and D.K.C. participated in fabrication of OLEDs. J.H.C. and C.M.L. contributed the fabrication of penicillin-based OLED devices. C.H.K. provided support and suggestions for TRPL and TRANES investigations. J.L., A.P., T.S.K., and J.K.S. prepared the ampicillin solution, characterized the HPLC and NMR studies, and provided theoretical support for ampicillin. H.J.Y., J.B.P., H.S.C., T.S.B., and S.G.L. helped to study the UPS/ XPS, AFM, HR-TEM, and XRD measurements, respectively. H.W.P. and K.B.C. recorded the ellipsometry studies. A.S. performed the KPFM analysis. J.H.K. delivered useful information about integrated sphere and the viewing angle measurements. H.W. Bae conducted the integral sphere measurements. Y.-C.K. and J.P. analyzed the N-1s profiles of pristine PEDOT:PSS using the XPS analysis. M.K.S. and C.S.K. provided valuable analysis and discussion.

\section{Competing interests}

The authors declare no competing interests.

\section{Additional information}

Supplementary information is available for this paper at https://doi.org/10.1038/s42005 019-0228-3.

Correspondence and requests for materials should be addressed to C.H.K. or S.Y.R.

Reprints and permission information is available at http://www.nature.com/reprints

Publisher's note Springer Nature remains neutral with regard to jurisdictional claims in published maps and institutional affiliations.

(c) (i) Open Access This article is licensed under a Creative Commons Attribution 4.0 International License, which permits use, sharing, adaptation, distribution and reproduction in any medium or format, as long as you give appropriate credit to the original author(s) and the source, provide a link to the Creative Commons license, and indicate if changes were made. The images or other third party material in this article are included in the article's Creative Commons license, unless indicated otherwise in a credit line to the material. If material is not included in the article's Creative Commons license and your intended use is not permitted by statutory regulation or exceeds the permitted use, you will need to obtain permission directly from the copyright holder. To view a copy of this license, visit http://creativecommons.org/ licenses/by/4.0/.

(C) The Author(s) 2019 